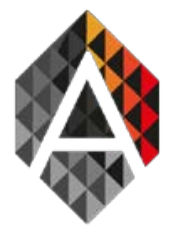

\title{
Enabling Communications in Heterogeneous Multi-Agent Systems: Electricity Markets Ontology
}

Gabriel Santos a, Tiago Pinto ${ }^{\text {a }}$, Zita Vale ${ }^{\text {a }}$, Isabel Praça ${ }^{\text {a }}$ and Hugo Morais a

${ }^{a}$ Research Group on Intelligent Engineering and Computing for Advanced Innovation and Development (GECAD), Institute of Engineering, Polytechnic of Porto (ISEP/IPP), Rua Dr. António Bernardino de Almeida, 431, 4200-072 Porto, Portugal

gajls@isep.ipp.pt; tmcfp@isep.ipp.pt; zav@isep.ipp.pt; icp@isep.ipp.pt, hugvm@isep.ipp.pt

KEYWORD

Electricity Markets; Multi-agent Systems; Ontologies; Semantic Interoperability

\section{ABSTRACT}

Electricity markets worldwide are complex and dynamic environments with very particular characteristics, resulting from their restructuring and evolution into regional and continental scales, along with the constant changes brought by the increasing necessity for an adequate integration of renewable energy sources. The rising complexity and unpredictability in electricity markets has increased the need for the intervenient entities in foreseeing market behaviour. Several modelling tools directed to the study of restructured wholesale electricity markets have emerged. However, they have a common limitation: the lack of interoperability between the various systems to allow the exchange of information and knowledge, to test different market models and to allow market players from different systems to interact in common market environments. This paper proposes the Electricity Markets Ontology, which integrates the essential necessary concepts related with electricity markets, while enabling an easier cooperation and adequate communication between related systems. Additionally, it can be extended and complemented according to the needs of other simulators and real systems in this area.

\section{Introduction}

The power sector has been completely revolutionized by the emergence of liberalized Electricity Markets (EM), aiming to improve the system's efficiency while offering economic solutions. Governments all over the world are trying to increase the use of renewable energy over the last few decades (Sharma et al., 2014). The sector's restructuring process brought out several challenges, requiring the transformation of the conceptual models that previously dominated the power sector (Sioshansi, 2013). The privatization, liberalization and international integration of previously nationally owned systems are some examples of the transformations that have been applied, along with the EM evolution into regional and continental scales, supporting transactions of huge amounts of electrical energy and enabling the efficient use of 
renewable based generation in places where it exceeds the local needs. (Sharma et al., 2014; Sioshansi, 2013).

The Pan-European EM is a reference case of this evolution, where the majority of European EM have joined together into a common day-ahead coupled EM composed by several countries (PCR, 2015; ACER, 2012b). The Price Coupling of Regions (PCR) project (PCR, 2014) developed EUPHEMIA (EUPHEMIA, 2016), the Multi-Regional Coupling algorithm which integrates the various European EM in a PanEuropean market. PCR is an initiative of seven European market operators, who have developed the procedures, redundant decentralized but interlinked IT systems and a single algorithm that calculates electricity prices, net import and export positions, and cross border electricity flows in one single run. Significant work has still to be done despite the several coupling initiatives that already have been realized (ACER, 2012b). The Cross-Border Intraday market integration which aims at implementing an Intraday target model ("an evolution of continuous intraday trading, to include intraday capacity recalculation, capacity pricing reflecting congestion and the capability to trade sophisticated products”) on all EU borders, is a still in progress roadmap (ACER, 2015; ACER, 2012a). Other examples evidence the transformation of national EM into Regional and Continental EM, such as the U.S., like California Independent System Operator (CAISO) (CAISO, 2016) and Midcontinent Independent System Operator (MISO) (MISO, 2016); and Brazil, since all regions are integrated in a joint EM (ONS, 2016). These markets can be considered as Continental EM due to these countries' size.

The EM restructuring made the market more complex and competitive, posing new challenges to its evolved entities, forcing them to rethink their behaviour and market strategies. The efficient operation of the market may bring potential benefits. The complex rules and regulations that define the market structure should not encourage strategic behaviours that might reduce market performance and lead to market power inefficiency (Shahidehpour et al., 2002). It becomes essential for professionals in this area to completely understand the markets' principles and how to evaluate their investments under such a competitive environment. Market players and regulators are very interested in foreseeing market behaviour: market players to understand the market's behaviour and operation to anticipate scenarios and define strategies, in order to maximize their profits; while regulators need to test rules and market architectures before being implemented to detect and avoid market inefficiencies (Meeus et al., 2005). Thus, EM became an attractive domain for software developers (Santos et al., 2015; Morais et al., 2014; Vrba et al., 2014; Cincotti and Gallo, 2013; Pinto et al., 2011; Li and Tesfatsion, 2009; Koritarov, 2004).

The use of simulation tools becomes decisive in order to study, analyse, and test different alternatives for markets' structure and evolution, giving entities decision support to address the new challenges. The main purpose of these tools is to deal with the constantly evolving reality of EM and grant actors with appropriate solutions to adapt themselves to the new reality, gaining experience to act in the context of a changing economic, financial, and regulatory environment. Market players aim to optimize their results (minimize costs if buying; or maximize profits if selling) and operators must ensure a competitive and transparent market in which no entity has significant market power (Shahidehpour et al., 2002).

EM simulators must be flexible in order to handle this complex and evolving reality, providing players with proper tools to adapt themselves to this dynamic reality and learn from experience. Several studies sustain that multi-agent systems (MAS) are suitable for the simulation of EM, with the adequate simulation capabilities, considering the complex interactions of the involved players (Santos et al., 2016; Pinto et al., 2015; Cincotti and Gallo, 2013; Koritarov, 2004). The facilitated inclusion of new models, market mechanisms, player types, and different types of interactions are some of the main advantages that multi- 
agent approaches provide (Santos et al., 2015). Some reference modelling tools have emerged in this domain, such as AMES (Agent-based Modelling of Electricity Systems) (Li and Tesfatsion, 2009), EMCAS (Electricity Market Complex Adaptive System) (Koritarov, 2004) and MASCEM (Multi-Agent Simulator of Competitive Electricity Markets) (Santos et al., 2016; Santos et al., 2015).

MASCEM (Santos et al., 2016; Santos et al., 2015) is a modelling and simulation tool developed for studying the complex restructured EM. It provides players with competitive advantage in the market by providing them with simulation and decision-support resources. A multi-agent architecture is used once market players are complex entities with particular characteristics and objectives, making their own decisions and interacting with each other. Experimental results demonstrate a cohesive market behaviour from the simulated environment (Pinto et al., 2016; Pinto et al., 2011).

Current tools are directed to the study of different EM mechanisms and to the analysis of the relationships between market entities, but they do not enable the interoperability with external systems. Simulators could gain significant added value by sharing their knowledge and market models with other agent societies. Such tools would provide the means for an actual improvement in current EM studies and development (Alvarado-Pérez et al., 2015; Frikha et al., 2015).

This article presents the Electricity Markets Ontology (EMO) designed to provide the means to achieve interoperability between EM simulation platforms. After this introductory section, Section 2 introduces the most relevant related work. Section 3 details the EMO, while in section 4 a case study based on real data from several European EM operators is demonstrated. The final conclusions are featured in section 5.

\section{Related work}

Multi-agent technology is being increasingly used to represent, model and simulate complex and dynamic environments such as the EM (Santos et al., 2016; Cincotti and Gallo, 2013; Pinto et al., 2011; Li and Tesfatsion, 2009; Koritarov, 2004). Each agent represents a physical or virtual entity acting according its perception of the environment, behaviour, profile and goals. Some of the most important characteristics of each agent are the autonomy, the perception of the other players' actions, and the use of artificial intelligence algorithms; turning the agent-based simulation platforms much more complex than standard distributed simulation tools (Ferber, 1999). Some of the main reasons why multi-agent technology is widely chosen as the best option for developing complex simulation tools for constantly evolving environments are the possibility of representing different entities as independent software agents with their own particular behaviour and objectives; and the opportunity for easily enlarging the represented models.

\subsection{Multi-agent simulators}

The Agent-based Modelling of Electricity Systems (AMES) (Li and Tesfatsion, 2009) is an open-source computational laboratory for the experimental study of wholesale power markets restructured in accordance with US Federal Energy Regulatory Commission (FERC)'s market design. To experimentally test the extent to which commonly used seller market power and market efficiency measures are informative for restructured wholesale power markets, AMES uses an agent-base test bed with strategically learning traders. It includes the independent system operator, load-serving entities and generation companies, distributed across the busses of the transmission grid. To update the action choice probabilities currently 
assigned to the supply offers in its action domain, each generation company agent uses stochastic reinforcement learning.

The Electricity Market Complex Adaptive System (EMCAS) (Koritarov, 2004) applies an agent based approach where the agents' strategies are based on learning and adaptation. The agents model the restructured EM heterogeneity, considering the generation, demand, transmission and distribution companies, independent system operators, consumers and regulators. EMCAS provides simulations in a time continuum ranging from hours to decades, including various market pools and bilateral contracts.

The Multi-Agent Simulator of Competitive Electricity Markets (MASCEM) (Santos et al., 2016; Santos et al., 2015) was developed with the goal of overcoming these challenges and overtake the limitations presented by the mentioned simulators. Section 2.2 overviews MASCEM in detail.

\subsection{MASCEM overview}

MASCEM - Multi-Agent Simulator of Competitive Electricity Markets (Santos et al., 2016; Santos et al., 2015) is a modelling and simulation tool developed with the aim of studying the operation of complex and competitive restructured EM. It models the main complex and dynamic market entities and their interactions. To support players' decisions in accordance with their characteristics and goals, medium/longterm gathering of data and experience is also considered.

Software agents in MASCEM represent the main entities and players involved in EM, such as: market operator, system operator, market players (consumers, producers and/or prosumers), and the aggregators. Figure 1 shows a general overview of MASCEM's multi-agent model.

Advances in Distributed Computing and

Artificial Intelligence Journal

(C)Ediciones Universidad de Salamanca / cc by-nc-nd
ADCAIJ, Regular Issue, Vol.5 N.2 (2016)

http://adcaij.usal.es 


\section{Aggregators}

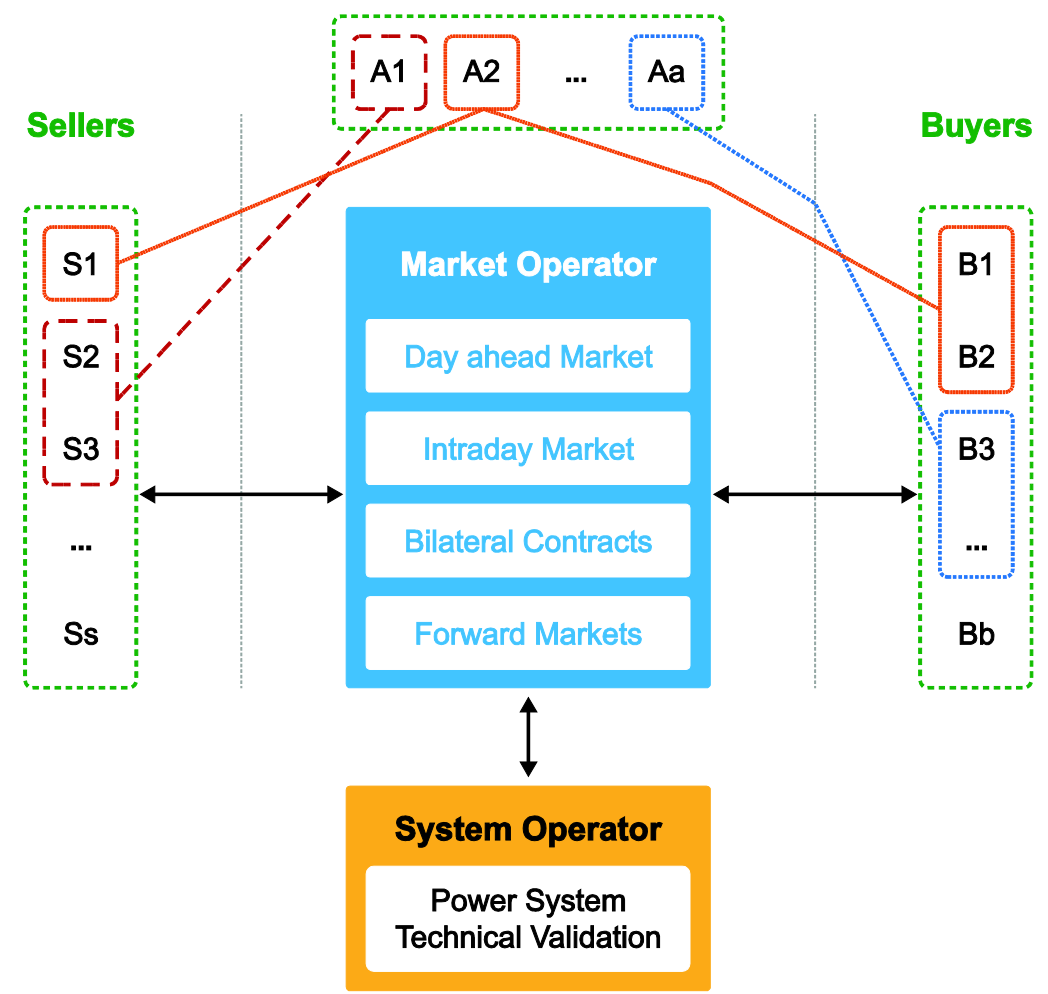

Figure 1: MASCEM's multi-agent model, adapted from (Santos et al., 2015)

The market operator agent is responsible for coordinating and regulating the pool markets operation. It informs buyer and seller agents when the pool is open, receives their proposals, validates and analyses them, and determines the clearing market price, accepted and refused bids for each trading period.

The system operator agent is responsible for the system's security and ensures that all constraints are satisfied within the system. It is always present during the simulations. After being informed by the market operator of the market's outcome, it examines the technical feasibility from the power system point of view and solves congestion problems that may arise.

Buyer and seller agents - the market players - are the key elements of EM. Buyer agents represent the demand side entities, while generation units are represented by seller agents. On one hand, sellers compete with each other trying to increase their profits; on the other, they may also cooperate with buyer agents trying to reach agreements that are advantageous for both parties.

The aggregators represent alliances of small independent players. The meaningful increase of small independent producers and consumers participating in the market, brought the need to make such alliances to enable them competing with big producers. They manage their aggregates' information and are seen in

Advances in Distributed Computing and

Artificial Intelligence Journal

(C)Ediciones Universidad de Salamanca / cc by-nc-nd
ADCAIJ, Regular Issue, Vol.5 N.2 (2016)

http://adcaij.usal.es 
the market as buyer or seller agents. Each aggregator is modelled as an independent MAS, allowing agents to be installed on different machines while maintaining the high performance as possible.

MASCEM includes the main types of negotiations normally present in EM, such as: day-ahead and intraday pool (symmetric or asymmetric, with or without complex conditions) markets; bilateral contracts and forward markets. By selecting a combination of these market models, it's also possible to perform hybrid simulations.

The user defines each scenario by inputting the market and market type to simulate, the number of simulation days, the number of participating players and their strategies considering each type of agent, with their own decision-support resources, assuring them competitive advantage in the market.

Communications between agents are carried out through the exchange of messages. The Foundation for Intelligent Physical Agents (FIPA) suggests Agent Communication Language (ACL) as a standard for communications between agents (FIPA, 2004). Its content includes the content language, specifying the syntax, and the ontology which provides the semantics of the message assuring the correct interpretation (FIPA, 2002). MASCEM agents use the Electricity Markets Ontology (EMO) to enable the interoperability with other MAS or agent-based simulators that intend to participate in MASCEM's simulations.

\section{Electricity Markets Ontology}

The integration of heterogeneous MAS raises inherent issues to the interconnection of those systems, particularly those involving the use of ontologies independently developed (Dai et al., 2013). These issues need to be addressed in the power industry in order to disseminate the development of interoperable MAS (McArthur et al., 2007). There is a growing need for knowledge exchange between these systems in order to take full advantage of their functionalities. The increasing application of multi-agent technology within power engineering promotes the adoption of standards that enable the communication between heterogeneous systems, bringing future advantages (Catterson et al., 2010). Open standards are needed to provide full interoperability.

Currently, MAS in the power system's domain are developed with their own specific ontologies. These systems share common concepts that are differently represented between the independently developed ontologies, and translating these concepts automatically is not as straightforward as it may seem. FIPA suggests the use of an OA, which provides some related services, to solve the problem of multiple ontologies (FIPA, 2001). This is still an experimental standard and mappings between ontologies still must be performed by ontologies' designers, which increases the human effort required and costs of implementation.

In alternative, Catterson (Catterson et al., 2010) proposes the use of an upper ontology representing the general concepts of the domain, ensuring a common basis for the representation of those concepts and their relationships between systems while reducing the complexity of ontology mapping. However, bearing in mind that applications must develop lower-level ontologies for all the application-specific concepts. According to Catterson (Catterson, 2006) there will be no need to modify existing agents when a new system is integrated, if the agents conform to a single upper ontology, as high level concepts would be universally understood, and only these concepts would be discussed. The disadvantage of this approach is that defining high level concepts is a very arduous and complex task, which requires a universal acceptance from all entities involved in the field. 
Rather than developing an "electricity markets and power systems upper ontology", as has been initially considered, this work proposes the development of ontologies for the interoperability of EM multi-agent simulation platforms, which can be extended in a way to enable the full interoperability between those systems. This is the case of the EMO.

\subsection{Existing ontologies within electricity markets’ domain}

To reuse existing ontologies is usually a requirement for systems' interoperability. It is almost always worthy to check if someone's work can be extended and refined for our particular domain or task. Even if the ontology is not expressed in the same formalism, knowledge representation systems are usually able to export and import ontologies and translate them from one formalism to another. There are some libraries of reusable ontologies available online, such as Ontolingua ${ }^{1}$ and $\mathrm{DAML}^{2}$ ontology libraries.

It is possible to find in the literature some ontologies developed for the field of energy markets, namely electricity and natural gas (Dam and Chapping, 2010; Dam and Keirstead, 2010; Alexopoulos et al., 2009). Unfortunately, none is publicly available for reuse and/or extension, which led to the development from scratch of the proposed ontology.

In (Alexopoulos et al., 2009) a very extensive and interesting work has been developed, although the authors decided to take in mind only the domain of the ontology, leaving aside its application scenario. It is also important to note that the developed ontology is aimed at the Greek EM, not having been extended to any other European EM. If this ontology was publicly available, its reuse would be considered, given the extensity and accuracy of the knowledge already represented. In any case, ELMO ontology (Alexopoulos et al., 2009), as it is, is not suitable for EM multi-agent simulators.

\subsection{Electricity Markets Ontology}

The EMO incorporates abstract concepts and axioms referring to the main existing EM. This ontology aims to be as inclusive as possible so that it can be extended and reused in the development of (lower level) market-specific ontologies, such as the MIBEL (MIBEL, 2016), EPEX (EPEXSPOT, 2016a), Nord Pool (NordPool, 2016b) or any other EM ontology. EMO is the base ontology from which all the remaining ontologies developed in the scope of this work are extended. This is also the ontology to extend to define a new market ontology, such as GME (the Italian electricity market) (GME, 2016) to be included in MASCEM or in other simulators.

After determining the domain and scope of the ontology, terms that are transversal to the various EM were identified in order to define the basic concepts for this domain. Figure 2 presents the main EM concepts identified and defined as classes in EMO.

\footnotetext{
${ }^{1}$ http://www.ksl.stanford.edu/software/ontolingua/

${ }^{2}$ http://www.daml.org/ontologies/
} 


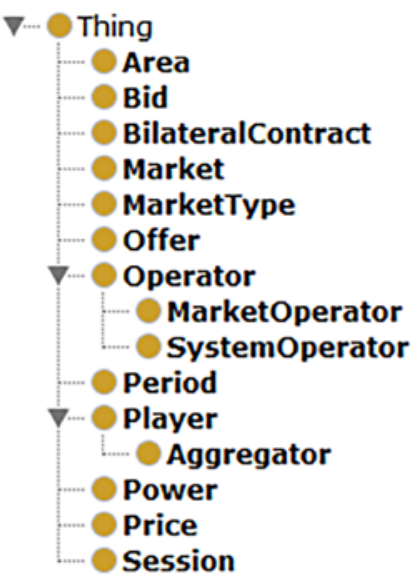

Figure 2: Electricity Markets Ontology classes

As it is possible to observe from Figure 2, EMO was kept as simple as possible in order to facilitate its reuse and extension independently of the market's features and/or rules. Figure 2, thereby identifies the main concepts transversal to global EM. However, given that the suggested ontologies were developed considering its use by agent based simulation tools, some markets' constraints were also defined in EMO, making use of data and object properties, assuring the expected behaviour in newly developed ontologies that import EMO. Figure 3 illustrates the object and data properties defined in EMO.

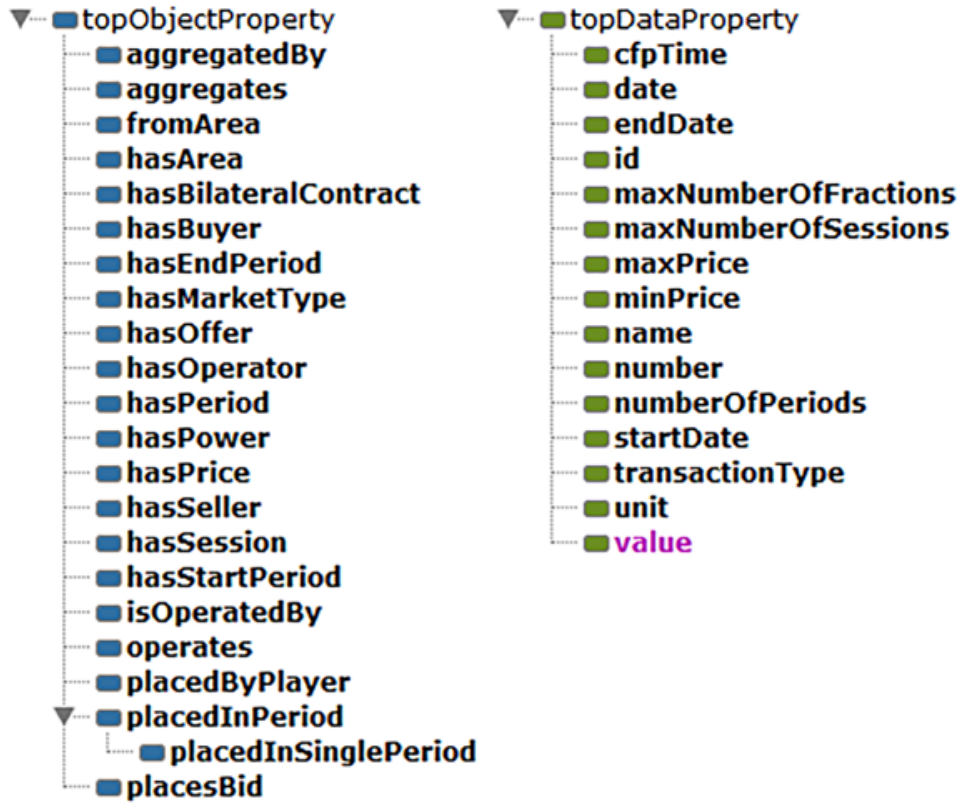

Figure 3: Electricity Markets Ontology object and data properties

Advances in Distributed Computing and

Artificial Intelligence Journal

(C)Ediciones Universidad de Salamanca / cc by-nc-nd
ADCAIJ, Regular Issue, Vol.5 N.2 (2016)

http://adcaij.usal.es 
An Object Property, in Protégé, is a property that relates two Objects (i.e. Classes), while a Data Property is a property relating an Object to a literal value (e.g. string, integer, etc.). In Figure 3 the object properties are represented in blue and the data properties in green. Relations between the identified classes and the object and data properties of EMO are illustrated in Figure 4.

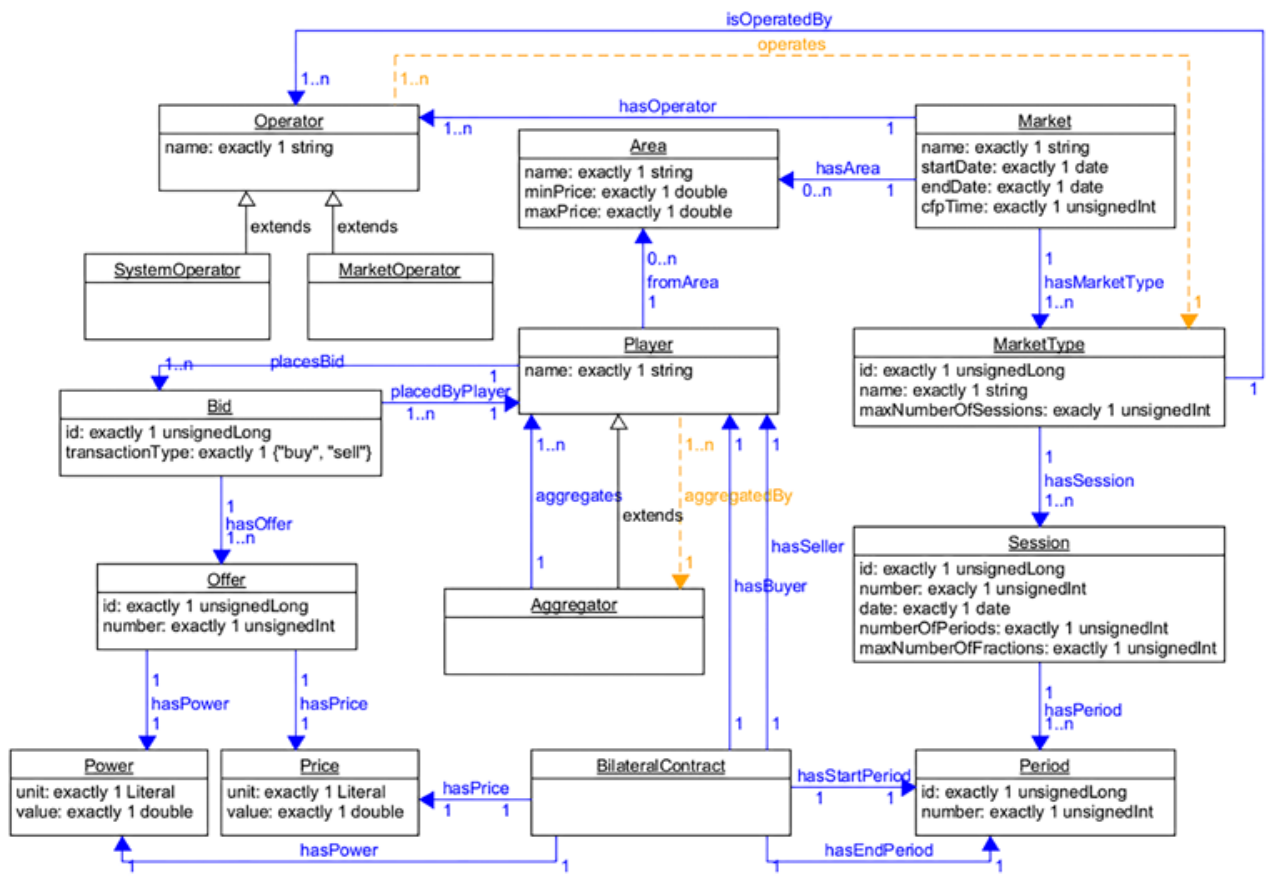

Figure 4: Electricity Markets Ontology

From Figure 4 it is possible to see the object properties represented in blue and the data properties defined within each class with the respective data types. The orange relations represent the inferred object properties, which are inverse properties of the ones defined in blue in the opposite direction. It should be noticed that three object properties defined in this ontology are not present in the UML diagram, namely: hasBilateralContract, placedInPeriod and placedInSinglePeriod. These are important properties that are introduced in EMO to be reused by the ontologies defined by each EM's domain.

The EMO has expressivity $A L C H I Q(D)$ and is defined in description logic (DL) syntax ${ }^{3}$. Table 3.1 presents the object properties, Table 3.2 the data properties and Table 3.3 the classes. The greater the number and variety of concepts that an ontology may represent, the greater is its expressiveness. The $A L$ (Attributive Language) is the base language allowing: (i) atomic negation, i.e. the negation of concept names that do not appear on the left side of axioms; (ii) concept intersection; (iii) universal restrictions; and

\footnotetext{
${ }^{3}$ http://www.obitko.com/tutorials/ontologies-semantic-web/owl-dl-semantics.html
} 
(iv) limited existential quantification. $C$ is the Complex concept negation extension. The $H$ extension is related with the role Hierarchy (e.g. the sub properties). The I extension represents the Inverse properties. The $Q$ extension are the Qualified cardinality restrictions, i.e. cardinality restrictions with fillers other than $\mathrm{T}$. And finally, the $(D)$ refers to the use of datatype properties, data values or data types.

\begin{tabular}{|c|c|}
\hline \multicolumn{2}{|c|}{ Object Properties } \\
\hline aggregatedBy $\sqsubseteq R$ & $\begin{array}{l}\text { hasPower } \sqsubseteq R \\
\mathrm{~T} \sqsubseteq \leq 1 \text { hasPower }\end{array}$ \\
\hline aggregates $\equiv$ aggregatedBy- & $\begin{array}{l}\text { hasPrice } \sqsubseteq R \\
\mathrm{~T} \sqsubseteq \leq 1 \text { hasPrice }\end{array}$ \\
\hline fromArea $\subseteq R$ & $\begin{array}{l}\text { hasSeller } \sqsubseteq R \\
\mathrm{~T} \sqsubseteq \leq 1 \text { hasSeller }\end{array}$ \\
\hline hasArea $\subseteq R$ & $\begin{array}{l}\text { hasSession } \sqsubseteq R \\
\mathrm{~T} \sqsubseteq \leq 1 \text { hasSession }^{-}\end{array}$ \\
\hline hasBilateralContract $\sqsubseteq R$ & $\begin{array}{l}\text { hasStartPeriod } \subseteq R \\
\mathrm{~T} \subseteq \leq 1 \text { hasStartPeriod }\end{array}$ \\
\hline $\begin{array}{l}\text { hasBuyer } \subseteq R \\
\mathrm{~T} \sqsubseteq \leq 1 \text { hasBuyer }\end{array}$ & isOperatedBy $\sqsubseteq R$ \\
\hline $\begin{array}{l}\text { hasEndPeriod } \subseteq R \\
\mathrm{~T} \sqsubseteq \leq 1 \text { hasEndPeriod }\end{array}$ & operates $\equiv$ isOperatedBy \\
\hline hasMarketType $\subseteq R$ & $\begin{array}{c}\text { placedByPlayer } \sqsubseteq R \\
\mathrm{~T} \sqsubseteq \leq 1 \text { placedByPlayer }\end{array}$ \\
\hline hasOffer $\sqsubseteq R$ & $\begin{array}{c}\text { placesBid } \equiv \text { placedByPlayer }^{-} \\
\quad \subseteq \leq 1 \text { placesBid }^{-}\end{array}$ \\
\hline hasOperator $\subseteq R$ & placedInPeriod $\sqsubseteq R$ \\
\hline $\begin{array}{l}\text { hasPeriod } \subseteq R \\
\mathrm{~T} \sqsubseteq \leq 1 \text { hasPeriod }^{-}\end{array}$ & $\begin{array}{c}\text { placedInSinglePeriod } \subseteq \text { placedInPeriod } \\
\quad T \sqsubseteq \leq 1 \text { placedInSinglePeriod }\end{array}$ \\
\hline
\end{tabular}




\begin{tabular}{|c|c|}
\hline \multicolumn{2}{|c|}{ Data Properties } \\
\hline cfpTime $\sqsubseteq U$ & name $\sqsubseteq U$ \\
\hline $\mathrm{T} \sqsubseteq \leq 1$ cfpTime & $\mathrm{T} \sqsubseteq \leq 1$ name \\
\hline date $\sqsubseteq U$ & number $\sqsubseteq U$ \\
\hline $\mathrm{T} \sqsubseteq \leq 1$ date & $T \subseteq \leq 1$ number \\
\hline endDate $\sqsubseteq U$ & numberOfPeriods $\sqsubseteq U$ \\
\hline \multicolumn{2}{|l|}{$\mathrm{T} \sqsubseteq \leq 1$ endDate } \\
\hline id $\sqsubseteq U$ & startDate $\sqsubseteq U$ \\
\hline $\mathrm{T} \sqsubseteq \leq 1 \mathrm{id}$ & $\mathrm{T} \sqsubseteq \leq 1$ startDate \\
\hline \multirow[t]{2}{*}{ maxNumberOfFractions $\sqsubseteq U$} & transactionType $\sqsubseteq U$ \\
\hline & $\mathrm{T} \sqsubseteq \forall$ transactionType. $\{$ "buy”, "sell” $\}$ \\
\hline \multirow[t]{2}{*}{ maxNumberOfSessions $\sqsubseteq U$} & unit $\sqsubseteq U$ \\
\hline & $\mathrm{T} \sqsubseteq \leq 1$ unit \\
\hline maxPrice $\sqsubseteq U$ & value $\sqsubseteq U$ \\
\hline $\mathrm{T} \sqsubseteq \leq 1$ maxPrice & $\mathrm{T} \sqsubseteq \leq 1$ value \\
\hline minPrice $\sqsubseteq U$ & \\
\hline $\mathrm{T} \sqsubseteq \leq 1$ minPrice & \\
\hline
\end{tabular}

Table 2: Electricity Markets Ontology data properties DL syntax

\begin{tabular}{|c|}
\hline Classes \\
\hline Area $\sqsubseteq T \sqcap 1$ name $\Pi 1$ maxPrice $\sqcap 1$ minPrice \\
\hline Operator $\sqsubseteq$ Т $\sqcap 1$ name \\
\hline MarketOperator $\sqsubseteq$ Operator \\
\hline SystemOperator $\subseteq$ Operator \\
\hline Period $\sqsubseteq T \sqcap 1$ id $\sqcap 1$ number \\
\hline Power $\sqsubseteq T \sqcap 1$ unit $\sqcap 1$ value \\
\hline Price $\sqsubseteq T \sqcap 1$ unit $\sqcap 1$ value \\
\hline 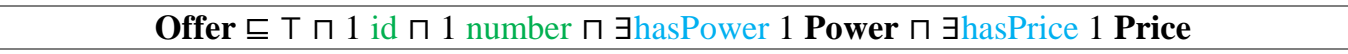 \\
\hline Player $\sqsubseteq \top \sqcap 1$ name $\Pi \exists$ fromArea Area $\Pi \exists$ placesBid Bid \\
\hline Aggregator $\subseteq$ Player $\sqcap \exists$ aggregates Player \\
\hline 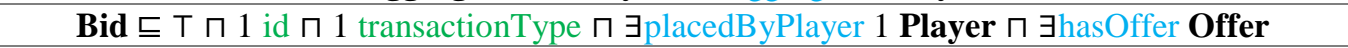 \\
\hline 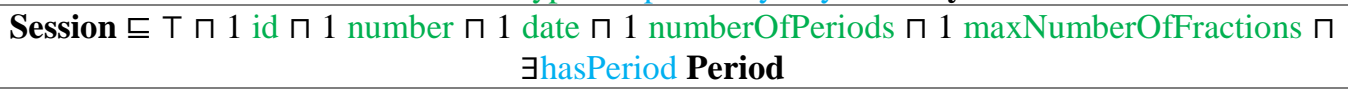 \\
\hline $\begin{array}{c}\text { MarketType } \sqsubseteq \top \sqcap 1 \text { id } \sqcap 1 \text { name } \Pi 1 \text { maxNumberOfSessions } \sqcap \text { ヨhasSession Session } \sqcap \\
\exists \text { isOperatedBy Operator }\end{array}$ \\
\hline 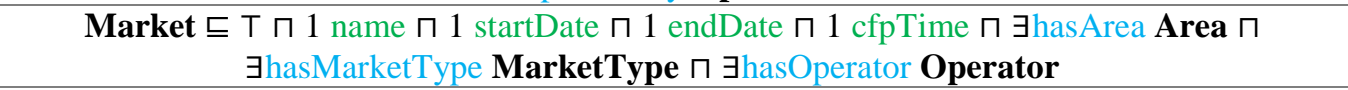 \\
\hline 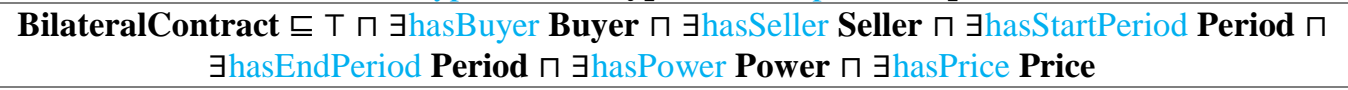 \\
\hline $\begin{array}{c}\text { Area } \sqcap \text { Operator } \sqcap \text { Period } \sqcap \text { Power } \sqcap \text { Price } \sqcap \text { Offer } \sqcap \text { Player } \sqcap \text { Bid } \sqcap \text { Session } \sqcap \text { Market } \sqcap \\
\text { MarketType } \sqcap \text { BilateralContract }=\perp\end{array}$ \\
\hline
\end{tabular}

Table 3: Electricity Markets Ontology classes DL syntax

Advances in Distributed Computing and Artificial Intelligence Journal

(C)Ediciones Universidad de Salamanca / cc by-nc-nd
ADCAIJ, Regular Issue, Vol.5 N.2 (2016) http://adcaij.usal.es 
The definition of an Area includes a string name, a double minPrice and a double maxPrice. All the three data properties are defined as Functional. A functional property is a property that only relates the same subject to one single object/value. Each EM area has an identifying name and its minimum and maximum prices are usually defined in its market rules.

An Operator includes only a name, while the MarketOperator and SystemOperator classes are extended from Operator. Other types of operators may be present in different EM, which can be defined is each market's ontology after importing the EMO.

A Period is here identified only with an id and (period) number. These two properties are both Functional as well, and it has been found important to include them in this ontology due to simulation and data storage purposes. It is certain that a period (of time) can also be defined with a start and end instants, but that terminology was left open so that, if required, one can always extend its definition in the ontology by importing EMO.

Both Price and Power are defined as a set of a unit (e.g. EUR and MW respectively) and a value in double, being these two data properties functional as well. An Offer, in turn, includes an id, a number and exactly a Power and a Price set by the object properties hasPower and hasPrice respectively. These two object properties are also Functional.

A Bid also includes an id, in addition to a transactionType ("buy" and "sell” only), a single Player (set with the Functional object property placedByPlayer) and Offers (set by the hasOffer object property).

A Player includes a name, and identifies its Area and placed Bids with the respective object properties fromArea and placesBid. The placesBid object property is the inverse of placedByPlayer, being also Inverse Functional, i.e. this property only relates the same object/value to a single subject. An Aggregator, on the other hand, is a subclass of Player, which aggregates other Players. The aggregates object property is inverse of the aggregatedBy object property, being this last inferred by the reasoner when active.

A Session includes an id, a number, a date, the numberOfPeriods and the maxNumberOfFractions data properties, and also the Periods. The date data property is Functional, the numberOfPeriods identifies the number of periods to consider in the simulation, while the maxNumberOfFractions determines the maximum number of fractions (Offers) per Bid. The Periods are set with the hasPeriod object property, which is Inverse Functional.

The MarketType is defined by an id, a name, the maxNumberOfSessions, including its Sessions and Operators. The maxNumberOfSessions determines the maximum number of sessions to consider in the simulation. The Sessions and Operators are set with the hasSession and isOperatedBy object properties respectively. The hasSession property is Inverse Functional and the isOperatedBy is the inverse of operates object property, which is inferred by the reasoner.

A Market comprises a name, a startDate, an endDate, a cfpTime, and its Area(s), MarketType(s) and Operator(s). The startDate, endDate and cfpTime properties are Functional. The startDate and endDate describe the simulation start and end dates, from which are also determined the number of simulation days. The cfpTime sets the call for proposal time limit a MarketOperator will wait to receive the Players' proposals. The Area(s) are set through the hasArea property, the MarketType(s) by the hasMarketType property and the Operator(s) via the hasOperator object property.

A BilateralContract includes a buyer and a seller Player, a start and an end Period, a Power amount and a Price offer. The players are set by the hasBuyer and hasSeller Functional object properties. The start and end periods by the hasStartPeriod and hasEndPeriod properties respectively, where both are also Functional. And the hasPower and hasPrice properties set the Power and Price respectively. 
Finally, the Area, the Operator, the Period, the Power, the Price, the Offer, the Player, the Bid, the Session, the Market, the MarketType and the BilateralContract classes are all Disjoint Classes, meaning that none of these classes has members in common. In other words, an element cannot be an instance of more than one of these classes, or else it makes the ontology inconsistent.

The ontology has been formulated in OWL DL, using the Protégé ${ }^{4}$ tool, and their representation is in $\mathrm{RDF} / \mathrm{XML}$. It is publicly available in MASCEM's website ${ }^{5}$ so that it can be used by third-party developers who wish to integrate their agent-based simulators with MASCEM, taking advantage of its simulation capabilities and market models. On the other hand, the ontology may also be reused and extended for the development of new multi-agent simulation tools in the context of wholesale EM.

\subsubsection{Additional modules}

To enable semantic communication between the market operator and player agents, two additional modules have been developed separately from EMO. These are: (i) the Call For Proposal Ontology (CFP) and (ii) the Electricity Markets Results Ontology (EMR). Although these are not detailed in this paper, are also publicly available ${ }^{6}$.

The CFP has the purpose of being used by the market operator agents to ask player agents for bids to be placed in the market, and for players to send their proposals to the respective market operators. In turn, the EMR is the ontology used by market operator agents to inform player agents about their results and outcomes in the market.

Both modules have expressivity $A L C H I Q(D)$, similarly to EMO, and have also been formulated in OWL $\mathrm{DL}$, being represented in RDF/XML as well.

\section{Case Study}

This case study intends to demonstrate the usefulness and advantage of using EMO to support players' participation in the market. It is based on four scenarios using real data extracted from several European market operators. These scenarios have been created with the intention of representing the European reality through a summarized group of players, representing buyer and seller entities of each area of each regional market (e.g. in the Iberian market, each area represents a country: Portugal and Spain; while in other regional markets each area represents a different zone, such as several parts of different countries, e.g. Nord Pool; or several parts of the same country, e.g. GME).

The simulation scenarios include two agents for each area (a seller and a buyer), practicing the average prices and negotiating the total amount of power that have been transacted in each of these areas in the reality, for each of the considered simulation days. Forty-one areas are considered, i.e. 41 buyers and 41 sellers, resulting in a total of 82 players for these simulation scenarios.

\footnotetext{
${ }^{4}$ http://protege.stanford.edu/

${ }^{5} \mathrm{http}: / /$ www.mascem.gecad.isep.ipp.pt/ontologies/electricity-markets.owl

${ }^{6} \mathrm{http}: / /$ www.mascem.gecad.isep.ipp.pt/ontologies/call-for-proposal.owl, http://www.mascem.gecad.isep.ipp.pt/ontologies/electricity-markets-results.owl
} 
The four selected scenarios consider two days during the summer, and other two during the winter; and for each, one business day (Wednesday) and one weekend day (Sunday). The selected days were: 25th July, 2012 (Wednesday); 29th July, 2012 (Sunday); 16th January, 2013 (Wednesday); and 20th January, 2013 (Sunday). These dates have been selected because they represent regular days, concerning the transacted power volume and market price in each season.

The players, representing the entire European Continent, negotiate in a common market environment. Three market mechanisms are considered, all regarding day-ahead negotiations: the EPEX SPOT (EPEXSPOT, 2016b); the MIBEL spot market (OMIE, 2016); and Elspot market from Nord Pool (NordPool, 2016a). All players negotiate using each of these three market mechanisms, for each of the four days. Players' behaviour is assumed as if they are participating in their origin market, e.g. players from markets where complex conditions are permitted use the particularities of each of the other market mechanisms to transpose the condition as best as possible (a good example is the Indivisibility complex condition from MIBEL (OMIE, 2016; Santos et al., 2011), which can be easily replaced with a Elspot or EPEX SPOT block offer (EPEXSPOT, 2016b), (NordPool, 2016a), and vice versa).

The simulation begins with the market operators sending the call for proposals to all the registered players. Figures 5 show the call for proposals (CfP) sent by the Nord Pool market operator present in this simulation, for the day 25 $5^{\text {th }}$ July, 2012. 
<? xml version="1.0" encoding="UTF-8" standalone="no"?> $<$ rdf : RDF

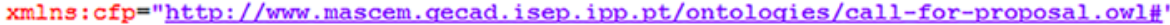

xmlns : emo="http://www. mascem. gecad. isep. ipp.pt/ontologies/electricity-markets.owl\#"

xmlns:nordpool="http://www. mascem.gecad. isep.ipp.pt/ontologies/nordpool. owl\#"

xmlns: owl="http://www.w3.ora/2002/07/owl\#"

xmlns: $r d f=$ "http://www .w3.orq/1999/02/22-rdf-syntax-ns\#"

xmlns: $r$ df $g="$ http://www.w3.orq/2000/01/rdf-schema\#"

xmlns: xsd="http://www.w3.orq/2001/xMLSchema\#"

日 xml:base="http://www.mascem.gecad.isep.ipp.pt/ontologies/">

南 〈rdf: Description rdf: about="nordpool.owl\# iM-NORDPOOL">

<emo:hasMarketType rdf:resource="nordpool.owl\#iMT-SPOT"/>

$<$ <emo: name>NORDPOOL</emo: name>

$\langle$ rdf : type rdf:resource="nordpool. owl\#NordPool" $/\rangle$

$</$ rdf: Description $>$

〈rdf: Description rdf: about="call-for-proposal.owl\#">

<owl: imports rdf: resource="nordpool. owl" "/>

<owl:imports rdf:resource="call-for-proposal. owl\#"/>

<rdf: type rdf: resource="http://www.w3.orq/2002/07/owl\#Ontolocy"/>

$</$ rdf : Description $>$

〈rdf: Description rdf : about="electricity-markets.owl\#iMO-NORDPOOL_2012_07_25"> <emo:name>NORDPOOL 201207 25</emo:name>

〈rdf: type rdf:resource="electricity-markets.owl\#Marketoperator"/> $\langle/$ rdf: Description $\rangle$

〈rdf: Description rdf : about="nordpool. owl\# iElspotSession2012-07-25-0"〉

〈rdf: type rdf:resource="nordpool. owl\#ElspotSession"/>

<emo:maxNumberofFractions rdf: datatype="

http://www.w3.ora/2001/XMLSchema\#unsianedInt" $>1</$ emo: maxNumberofFractions $>$

<emo: numberofPeriods rdf:datatype="http://www.w3.ora/2001/XMLSchemallunsianedInt"

$>24</$ emo: numberofPeriods $>$

<emo: number rdf :datatype="http://www.w3.ord/2001/XalLSchema\#nnsianedInt">0

$</$ emo: number $>$

<emo: id rdf : datatype="http://www.w3.ord/2001/XMLSchema\#nnsianedLong">

$6768671844112679792</$ emo: $i d>$

<emo: date rdf: datatype="http://www.w3.ord/2001/XMLSchema\#date" $>2012-07-25$

$</$ emo:date $>$

$</$ rdf: Description $>$

〈rdf : Description rdf : about="nordpool. owl\#iMT-SPOT">

<rdf: type rdf: resource="nordpool. owl\#Elspot"/>

<emo:isOperatedBy rdf:resource="electricity-markets.owl\#iMO-NORDPOOL_2012_07_25" />

<emo: hasSession rdf: resource="nordpool. owl\#iElspotSession2012-07-25-0"/>

$<$ <emo: name>SPOT</emo: name>

<emo:id rdf : datatype="http://www.w3.ord/2001/XMLSchema\#nsiqnedLong"> $5028184771267710553</$ emo: $i d>$

$\langle/$ rdf: Description $>$

〈rdf: Description rdf : about="call-for-proposal. owl\#iCFP-ElspotSession2012-07-25-0"〉

〈cfp:forElectricityMarket rdf: resource="nordpool. owl\#iM-NORDPOOL"/>

〈rdf: type rdf: resource="call-for-proposal. owl\#CallForProposal"/>

$</$ rdf: Description $>$

$-\langle/$ rdf : RDF $>$

Figure 5: Nord Pool Market Operator's CfP RDF

Advances in Distributed Computing and

Artificial Intelligence Journal

(C)Ediciones Universidad de Salamanca / cc by-nc-nd
ADCAIJ, Regular Issue, Vol.5 N.2 (2016)

http://adcaij.usal.es 
Analysing the CfP above, it is possible to check from line 40 to line 43 the definition of a CallForProposal for the electricity market named "NORDPOOL" (defined from line 11 to 15), with market type "SPOT" (between lines 33 and 39) and session "ElspotSession” (from line 25 to 32). After receiving the CfP, each player queries its KB in order to send its proposal to the respective market operator. Figure 6, Figure 7 and Figure 8 present examples of the market proposals sent by Seller 22 to each of the market operators, for the same day. The complete versions are available online ${ }^{7}$, where the prices and amount of power to trade in each market is more easily perceptible.

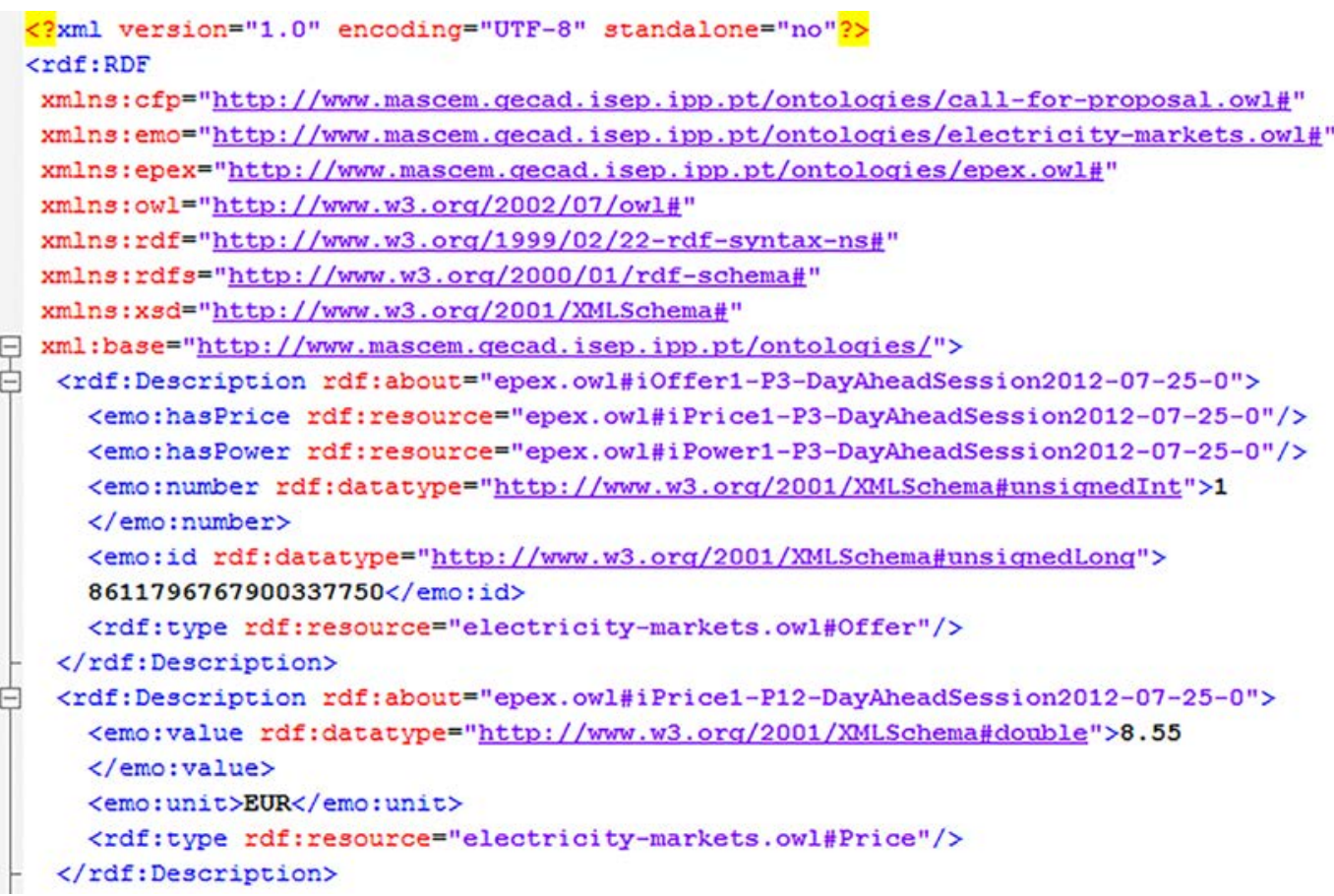

Figure 6: Proposal presented by Seller 22 to EPEX

\footnotetext{
${ }^{7}$ http://www.mascem.gecad.isep.ipp.pt/ontologies/case-study/1/\#rdf-4 http://www.mascem.gecad.isep.ipp.pt/ontologies/case-study/1/\#rdf-5 http://www.mascem.gecad.isep.ipp.pt/ontologies/case-study/1/\#rdf-6
}

Advances in Distributed Computing and Artificial Intelligence Journal

(C)Ediciones Universidad de Salamanca / cc by-nc-nd
ADCAIJ, Regular Issue, Vol.5 N.2 (2016)

http://adcaij.usal.es 


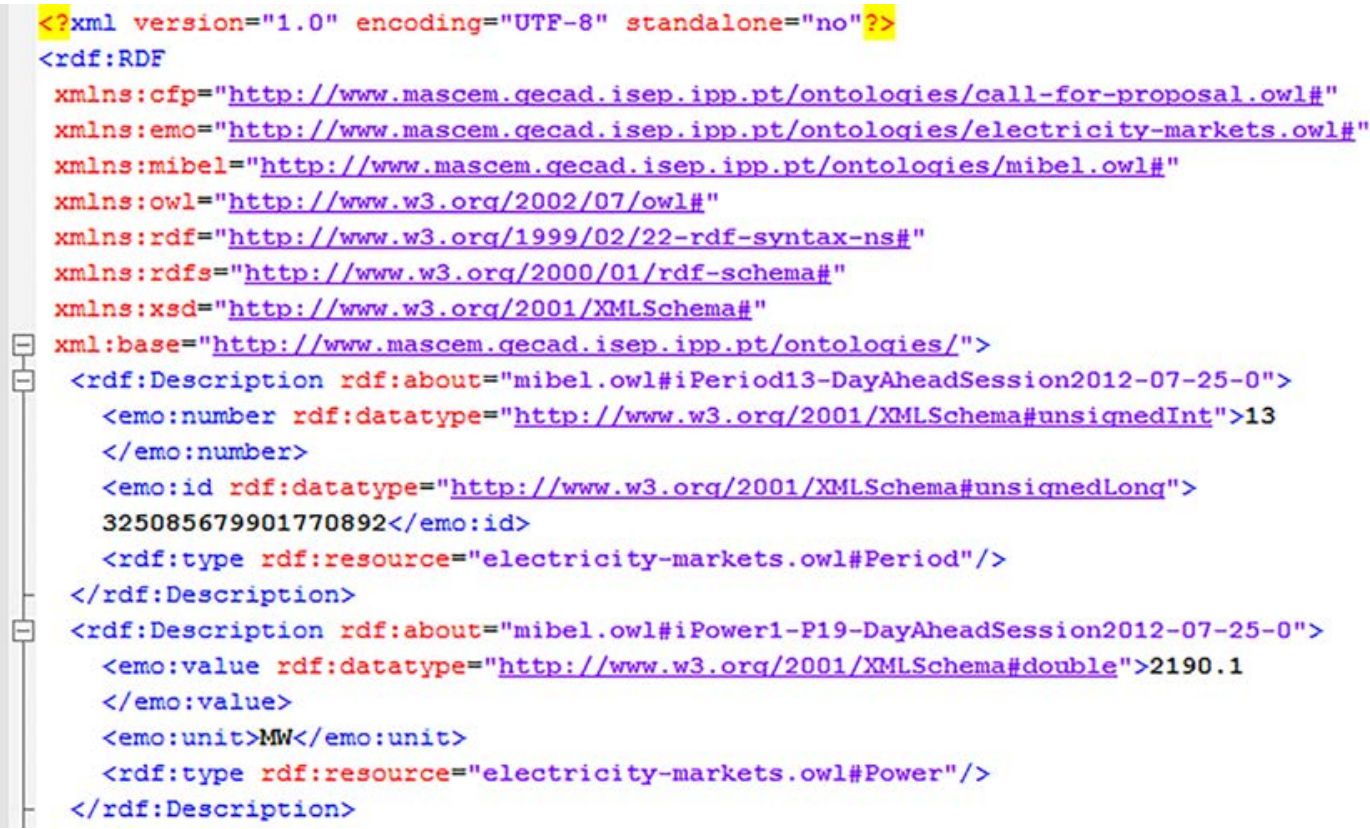

Figure 7: Proposal presented by Seller 22 to MIBEL

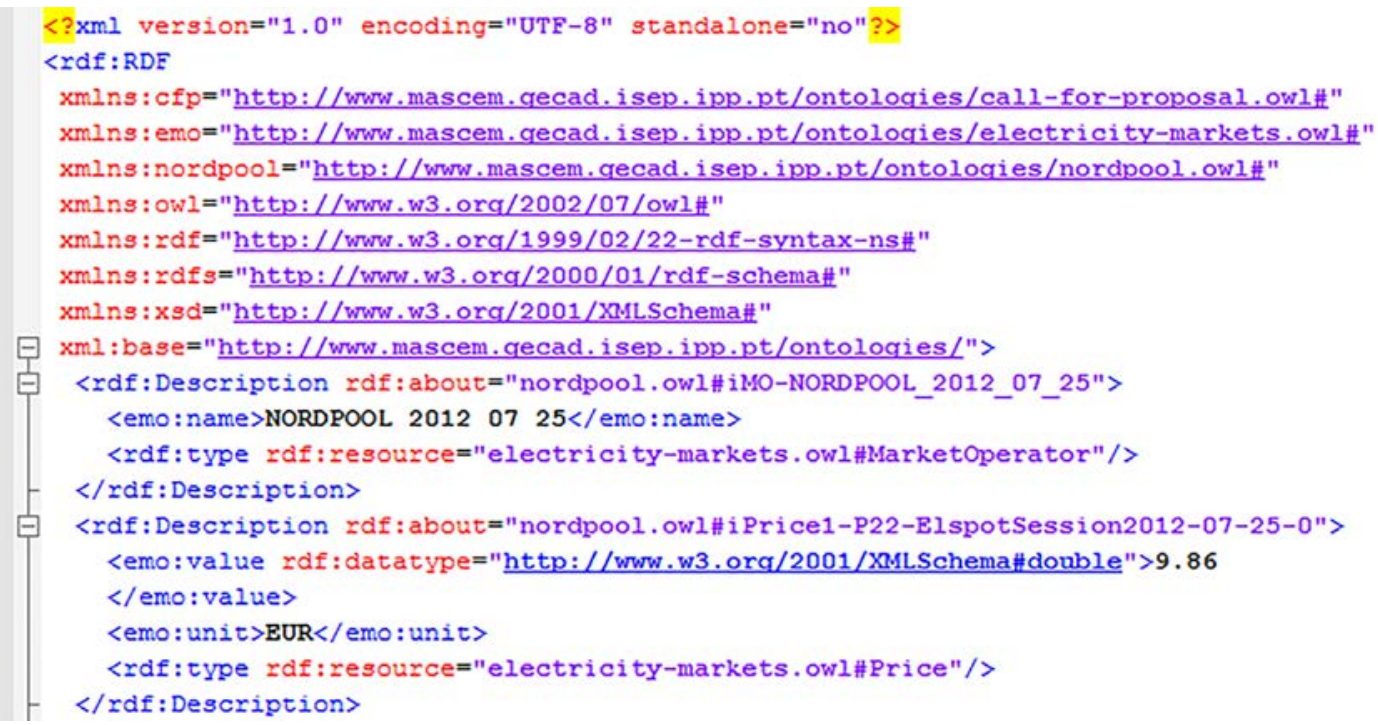

Figure 8: Proposal presented by Seller 22 to Nord Pool

After receiving the proposals and validating all incoming offers, each market operator analyses the bids, and generates the RDF results to be sent to the participating players. The RDF results achieved by Seller 
22 in each of the markets for the day 25 $5^{\text {th }}$ July, 2012 are illustrated in Figure 9, Figure 10 and Figure 11. The full version of these RDF can be found online ${ }^{8}$, where the results may be observed with better insight.

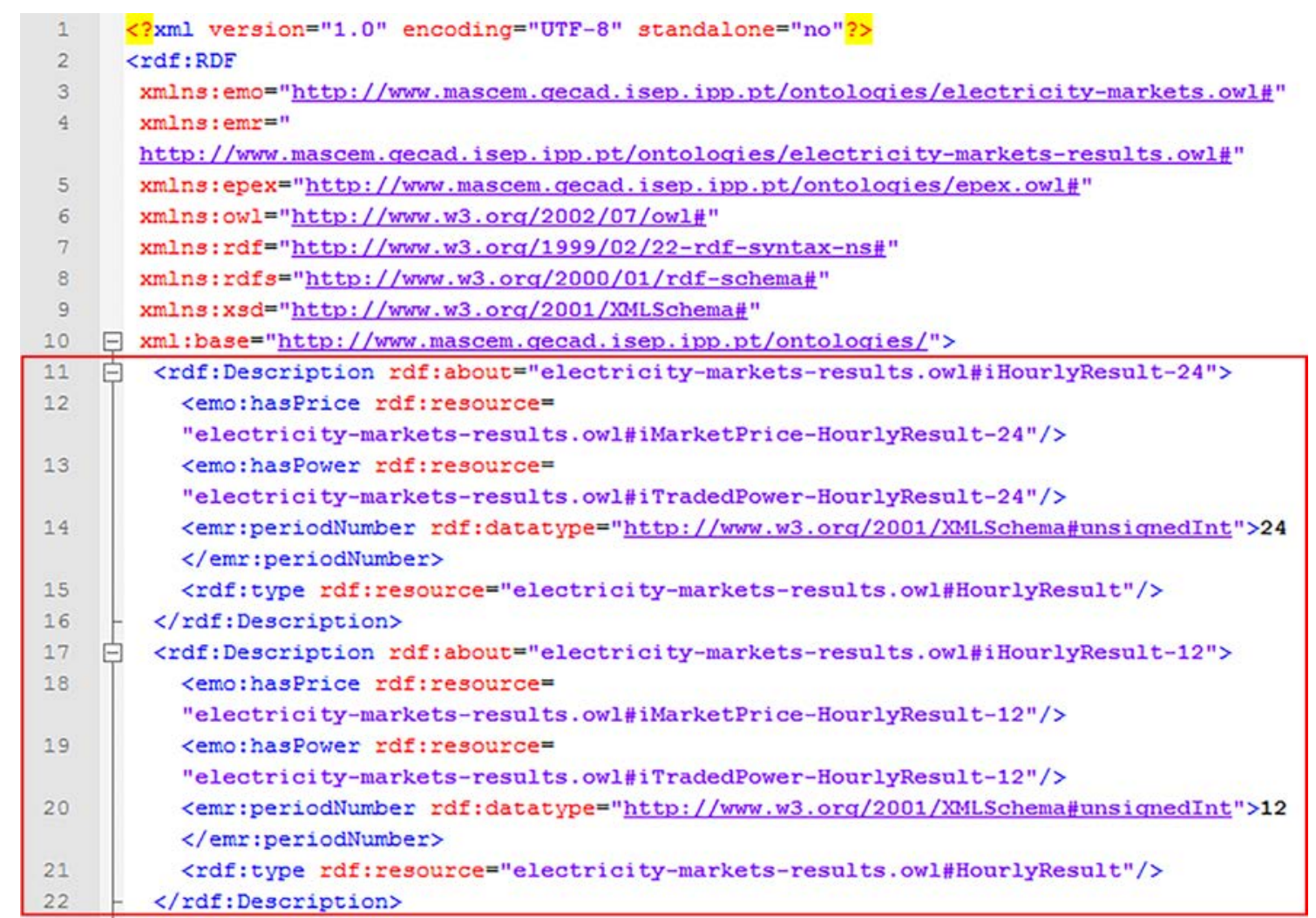

Figure 9: Results achieved by Seller 22 on EPEX

${ }^{8} \mathrm{http}: / /$ www.mascem.gecad.isep.ipp.pt/ontologies/case-study/1/\#rdf-7 http://www.mascem.gecad.isep.ipp.pt/ontologies/case-study/1/\#rdf-8 http://www.mascem.gecad.isep.ipp.pt/ontologies/case-study/1/\#rdf-9

Advances in Distributed Computing and Artificial Intelligence Journal

(C) Ediciones Universidad de Salamanca / cc by-nc-nd
ADCAIJ, Regular Issue, Vol.5 N.2 (2016) http://adcaij.usal.es 
<? xml version="1.0" encoding="UTF-8" standalone="no"?>

$<$ rdf:RDF

xmlns : emo="http://www. mascem. gecad. isep. ipp.pt/ontologies/electricity-markets.owlll" xmlns : emr="

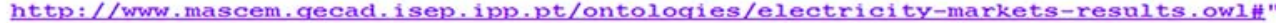

xmlns : mibel="http://www, mascem, gecad, isep, ipp.pt/ontologies/mibel. owl\#"

xmlns : owl = "http: //www.w3.ora/2002/07/owll"

xmlns : rdf ="http://www.w3.ord/1999/02/22-rdf-syntax-ns"

xmlns: $r d f s="$ http: //www.w3.ora/2000/01/rdf-schemall"

xmlns : xsd="http://www.w3.ord/2001/XMLSchemall"

日 xml :base="http://www.mascem. gecad.isep.ipp.pt/ontoloqies/">

<rdf: Description rdf:about="electricity-markets-results.owl\#iHourlyResult-24"> <emo: hasprice rdf:resource=

"electricity-markets-results.owl\#iMarketPrice-HourlyResult-24"/>

<emo:haspower rdf: resource=

"electricity-markets-results. owl"i TradedPower-HourlyResult-24"/>

<emr:periodNumber rdf : datatype="http://www.w3.ora/2001/xalLSchemallunsianedInt" $>24$ $</$ emr:periodNumber>

<rdf:type rdf:resource="electricity-markets-results.owl\#HourlyResult"/>

$\langle/$ rdf : Description $>$

<rdf: Description rdf: about="electricity-markets-results.owl\#iHourlyResult-12">

<emo:hasprice rdf: resource=

"electricity-markets-results.owl\#iMarketPrice-HourlyResult-12"/>

<emo: haspower rdf:resource=

"electricity-markets-results. owl" iTradedPower-HourlyResult-12"/>

<emr:periodNumber rdf: datatype="http://www. w3. ord/2001/xalLSchemallunsicmedInt" >12

$</$ emr:periodNumber $>$

<rdf: type rdf:resource="electricity-markets-results.owliHourlyResult"/>

$</$ de: Description $>$

Figure 10: Results achieved by Seller 22 on MIBEL

$\langle ? \mathrm{xml}$ vergion="1.0" encoding="UTF-8" standalone="no"?>

$<$ rdf : RDE

xmlns : emo="http://www.mascem.qecad.isep.ipp.pt/ontologies/electricity-markets.owl\#"

xmlns: emr="

http://www.mascem. gecad. isep.ipp.pt/ontologies/electricity-markets-results. owl\#"

xmlns: nordpool="http://www.mascem. gecad.isep.ipp.pt/ontoloqies/nordpool. owl\#"

xmlns: owl $="$ http: //www.w3.ora/2002/07/owl\#"

xmlng: rdf="http://www.w3,ord/1999/02/22-rdf-syntax-ns\#"

xmlns : rdf $g=$ "http://www.w3.orq/2000/01/rdf-schema\#"

xmlns : xsd="http://www.w3.orq/2001/xal.Schema\#"

Đ xml:base="http://www.mascem.gecad.isep.ipp.pt/ontologies/">

古 〈rdf: Description rdf:about="electricity-markets-results.owl\#iflexibleResult-2">

<emo:hasprice rdf:resource=

"electrici ty-markets-results. owl\#iMarketPrice-FlexibleResult-2"/>

<emo: haspower rdf: resource=

"electricity-markets-results.owl\#i TradedPower-FlexibleResult-2"/>

<emr:periodNumber rdf: datatype="http://www.w3.orq/2001/XMLSchema\#nnsianedInt" $>2$

$</ e m r$ :periodNumber $>$

<rdf: type rdf:resource="electricity-markets-results.owl\#FlexibleResult"/>

$</$ rdf: Description>

<rdf: Description rdf:about=

"electricity-markets-results.owl\#i TradedPower-FlexibleResult-11">

<emo: unit>MW</emo:unit>

<emo:value rdf:datatype="http://www.w3.ord/2001/XMLSchema\#double" $>0.0</$ emo:value>

<rdf:type rdf:resource="electricity-markets-results.owl"TradedPower"/>

$</$ rdf : Description $>$

Figure 11: Results achieved by Seller 22 on Nord Pool

Advances in Distributed Computing and

Artificial Intelligence Journal

(C)Ediciones Universidad de Salamanca / cc by-nc-nd
ADCAIJ, Regular Issue, Vol.5 N.2 (2016)

http://adcaij.usal.es 
Figure 9 and Figure 10 present hourly results for periods 12 and 24 (between lines 11 and 22), while Figure 11 shows the flexible result achieved by Seller 22 on period 2 (line 11 to 16). Figure 12 presents the market results for each of the four considered days, using each of the three market types' mechanisms.
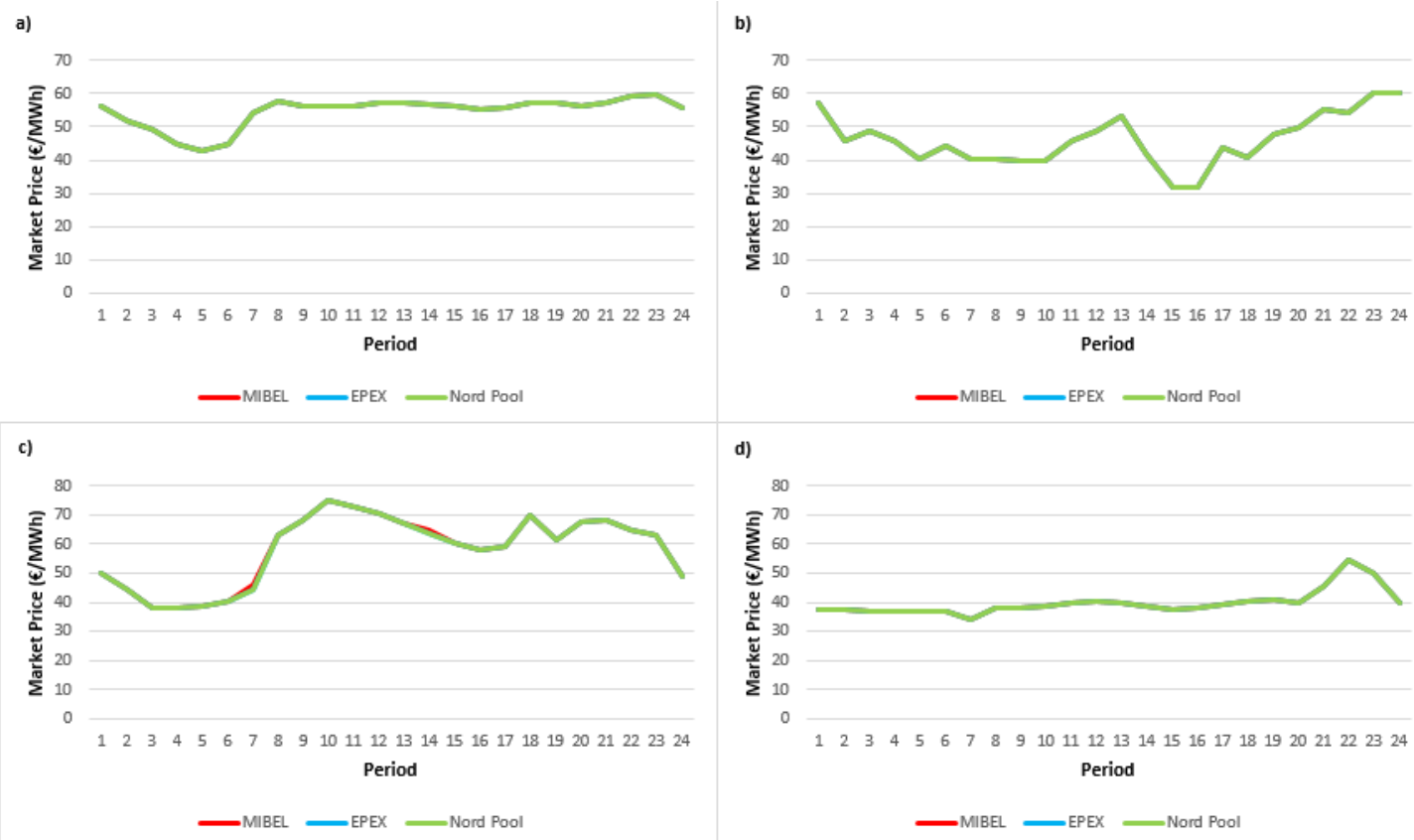

Figure 12: Electricity market prices for the conjunct participation of all European countries in: a) 25th July, 2012; b) 29th July, 2012; c) 16th January, 2013; d) 20th January, 2013.

From Figure 12 it visible that the market prices are almost the same, every day, when using the three market mechanisms. In the case of $16^{\text {th }}$ January, 2013, show in Figure 12 c) the market prices in MIBEL in periods 7 and 14 differ slightly from the other.

These small differences are due to the fact that, in spite of the three market mechanisms being based on a symmetric auction (i.e. the basis of the markets is identical), all markets present particularities that distinguishes them. The possibility of presenting complex conditions, block offers and flexible offers, gives the participant players the chance to adapt their behaviour to the specificities of each market. This means that the way players act in each market has a direct influence on the outcomes of the market, therefore the use of simulation tools, which allow them to test new approaches in order to learn how to act in a new environment, is a critical issue.

Regarding the seasonal differences, when comparing the four simulated days, a small decrease in the prices is verified from summer to winter. This decrease is also visible in all markets during the weekend days when compared to the business days of the same season.

Considering the results of each player in the three different EM, it is possible to determine the social welfare (SW) to evaluate the global players' benefits in each one. The SW is calculated as in (1), following the principles presented in (Wu et al., 2008). 


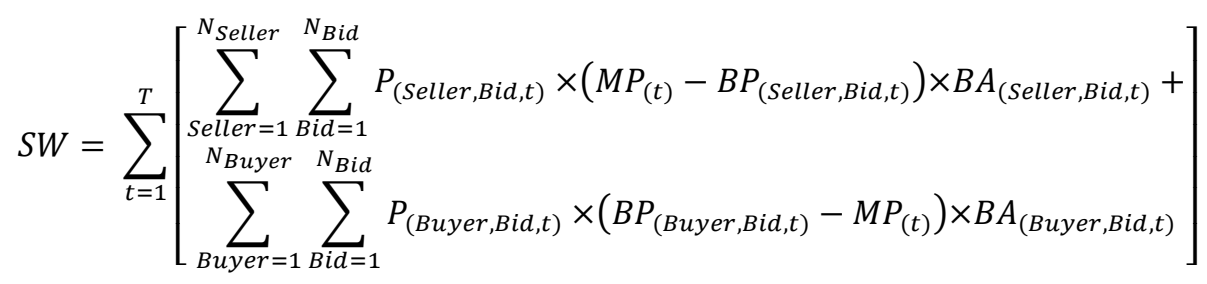

where $P_{(\text {Player,Bid,t })}$ and $B P_{(\text {Player,Bid,t) }}$ are the power and price offered by the player (Seller or Buyer) in a specific bid for period $t$, respectively. $M P_{(t)}$ is the market clearing price in period $t$ and $B A_{(\text {Player }, B i d, t)}$ is a binary variable which indicate if the bid for period $t$ was accepted. The results are presented in Figure 13.

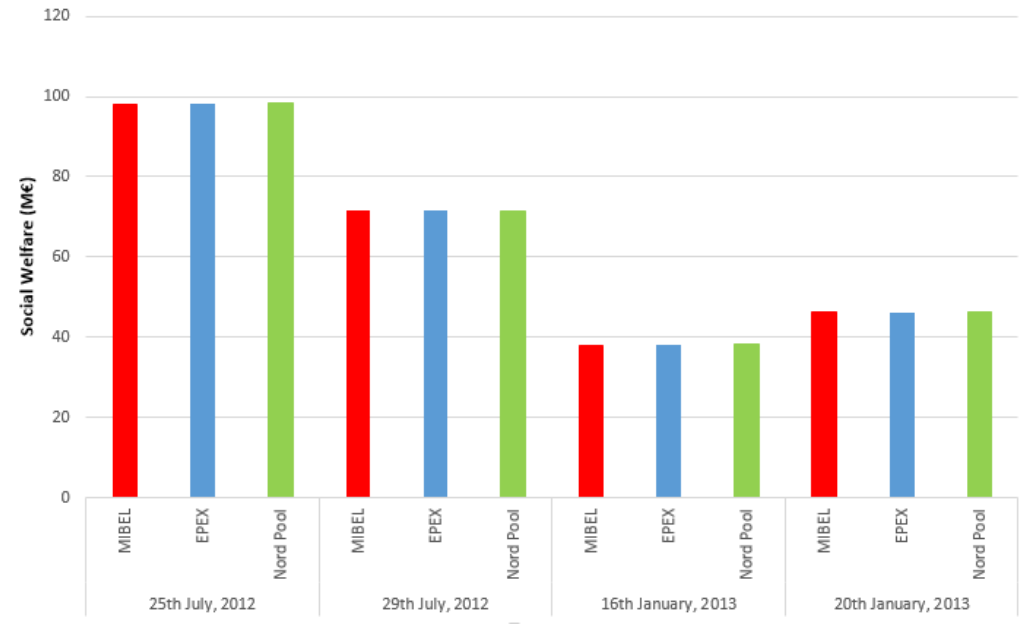

Figure 13. Social welfare

As it is possible to see in Figure 13, the SW is similar in the three markets. In all days, the Nord Pool presents a slightly higher SW, with an average difference of $0.3 \%$. The differences of SW are marginal for the different markets in the same day because all the markets use a symmetric clearing mechanism. Additionally, the values represent the sum of the SW of all periods, absorbing the differences that may exist in individual periods. In contrast, the SW changes significantly in different days due to the differences in the demand requirements and mainly in the resources (production) availability.

In order to illustrate the impact of using different types of offers, available in the different markets, the outcomes of one particular seller (Seller 22) are analysed, when participating in the three different market mechanisms, for the day of $25^{\text {th }}$ July, 2012. This player uses the proposed ontology to participate in the different market mechanisms while maintaining, as possible, its strategic approach for market negotiations.

Figure 14 presents the results of Seller 22 during the daily market session, using the MIBEL market mechanism. This player uses the Indivisibility complex condition, to ensure that the whole production amount is sold. 


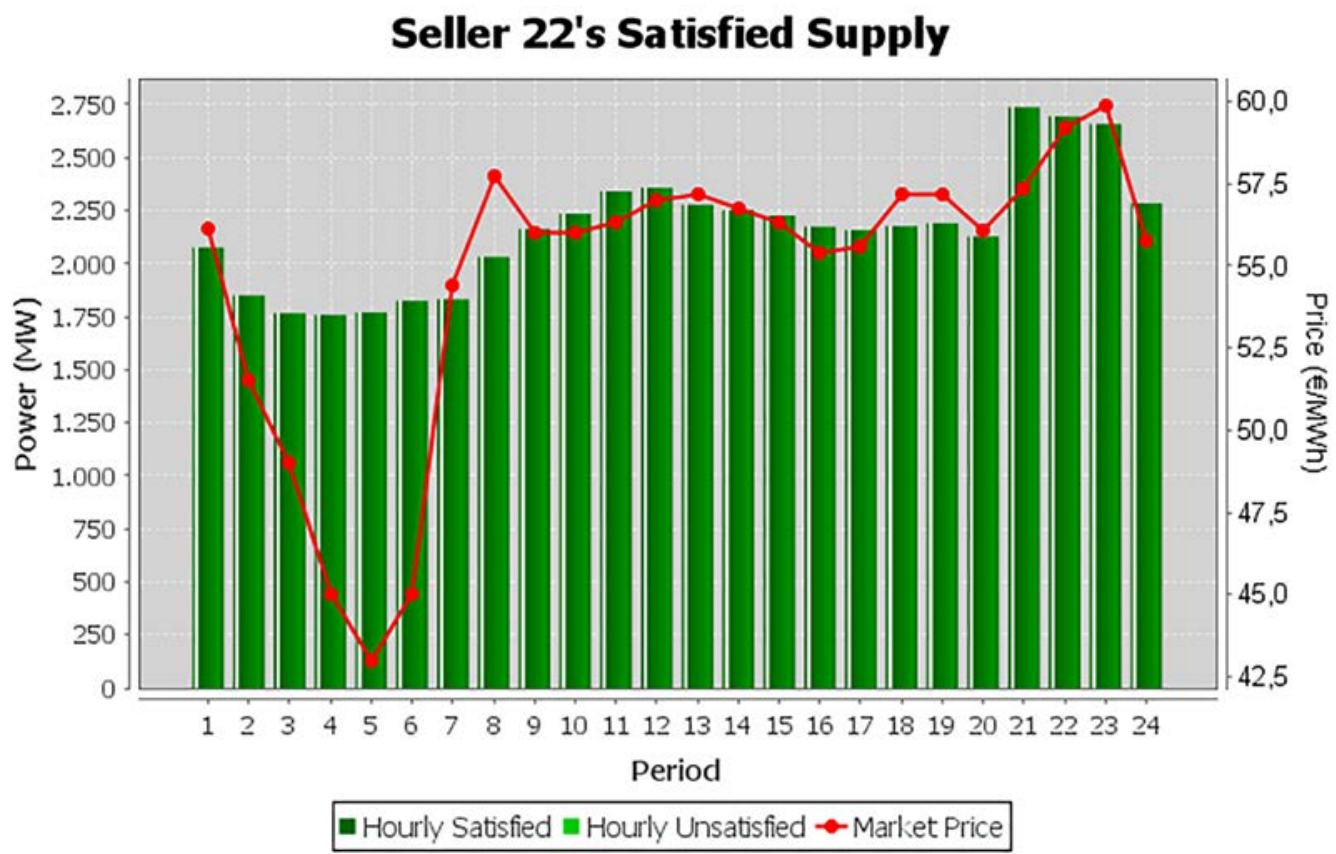

Figure 14: Market results of Seller 22 when participating in the MIBEL market mechanism, with the Indivisibility complex condition.

Given that Seller 22 needs to sell all of its available energy, the bid price that this player submitted was very low when compared to the average, expected, market price. As a result, all of the energy available was indeed sold in the market, as it is possible to observe in Figure 14.

Figure 15 presents the market results for Seller 22, when participating in the EPEX spot market, using block offers. This type of offer can be seen as a group of single hourly offers, where each order can have a different amount of energy, but all have the same price. The orders included in the block must belong to three or more consecutive hours.

These orders have a fill-or-kill condition, which means that all of the orders must be accepted in the market for the block to be negotiated. If only block offers are used, it would be very similar to the use of the Indivisibility condition of MIBEL. The use of the agents' ontologies allows players to be aware of this. However, since the main objective of this player is to sell as much power as possible, Seller 22 offers the majority of its available power at low prices, but using the block offers trying to optimize the price on a smaller amount of power, assuming that risk.

The block order submitted by Seller 22 is composed of 24 individual orders, one for each of the 24 hourly periods of the market session. The same energy volume was defined for all of the orders (200 MWh) and the price set for the block is $44 € / M W h$. 


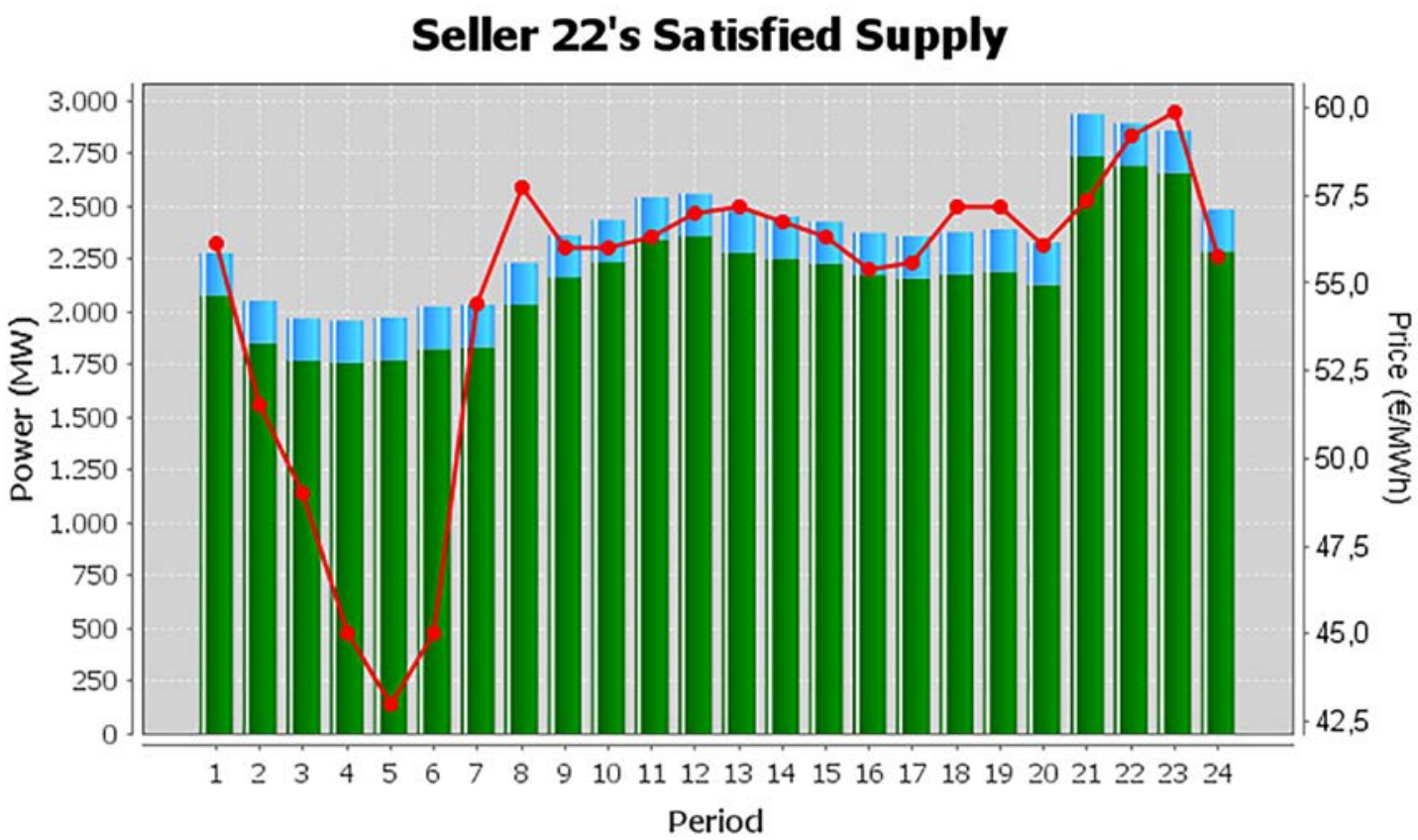

a Hourly Satisfied $\mathbf{m}$ Hourly Unsatisfied $\mathbf{m}$ Block Satisfied $\mathbf{m}$ Block Unsatisfied $\bullet$ Market Price

Figure 15: Market results of Seller 22 when participating in the EPEX market mechanism, using block orders.

It is possible to observe in Figure 15, that the block was not accepted, despite its price being lower than the market price on 23 of the 24 hourly periods. The market price of the $5^{\text {th }}$ period was set at $42 € / \mathrm{MWh}$ (below the block offer price), which caused the entire block being refused in the market, according with the fill-or-kill condition.

Finally, Figure 16 presents the results of Seller 22 when participating in the European Market scenario, using the Nord Pool - Elspot mechanism. In this market mechanism, Seller 22 uses three flexible hourly orders. These flexible hourly orders (available only to seller agents), allow the players to specify a fixed price and volume. The hour is not specified. The order will be accepted in the hour that optimizes the overall socioeconomic welfare of the market. A maximum of five flexible hourly orders is available per agent during a market session. In this scenario three orders were submitted with the volume of $2000 \mathrm{MWh}$ each, all at the same price of $40 € / \mathrm{MWh}$. 


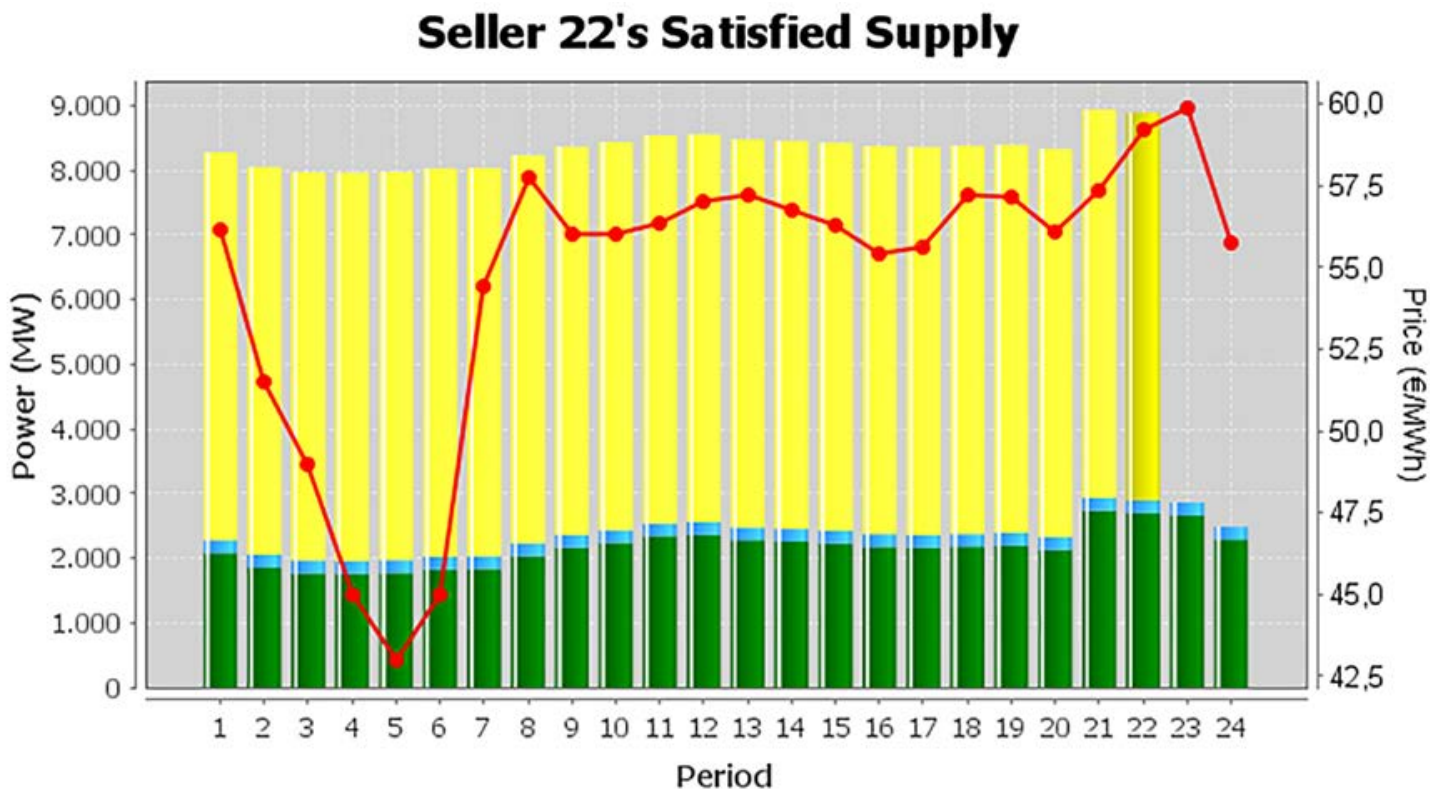

- Hourly Satisfied $\mathbf{m}$ Hourly Unsatisfied $\boldsymbol{m}$ Block Satisfied $\boldsymbol{m}$ Block Unsatisfied $=$ Flexible Satisfied

Flexible Unsatisfied - Market Price

Figure 16: Market results of Seller 22 when participating in the Elspot market mechanism, using block and flexible orders.

It is possible to observe from the chart of Figure 16 that during the first twenty-one periods (hours) none of the orders was accepted although bid price being below the established market price. The light yellow bars indicate a total of $6000 \mathrm{MWh}$ of unsold energy during these periods (referring to the total of the three flexible offers, of $2000 \mathrm{MWh}$ each). The flexible hourly orders were accepted in the $22^{\text {nd }}$ hourly period. In this period the total amount of energy of the order was sold.

As can be seen by the graph of Figure 16, the three flexible offers are accepted in period 22, being only the block orders unsatisfied in all of the 24 hourly periods. As mentioned before, the condition for the acceptance of each (or all) flexible offer(s) is not only the proposed bid price, but also the maximization of the overall socioeconomic welfare of the market session, from the market operator's perspective.

Comparing the results of Seller 22 when participating in each one of the three markets, it is possible to observe that it is vital for an agent to have a full understanding of all the different conditions that each market presents. The possibility of using different types of offers, such as complex conditions, flexible and block orders can make a colossal difference both in an individual player's profits, and also in the overall SW of the market. The flexible orders allow a player to sell an extra volume of energy, at a higher price, in hours when that energy is most demanded. By defining a lower price for a block order, a player can sell a predetermined amount of energy throughout the whole market session. In that case, the risk is not very high. However, if the player tries to maximize its profit, by setting a higher price, such as Seller 22 did 
when participating in the EPEX SPOT market, the risk of the whole block being rejected increases because of the fill-or-kill condition.

The use of the proposed ontology, which defines the characteristics and specifications of each different market, allows inferring market rules from the contained information. Taking these rules into account, behaviours can be modelled and adapted. In this case study, Seller 22 has used block orders in its participation in the EPEX SPOT market. These block orders are not available in MIBEL, however, the Indivisibility condition that is supported by MIBEL, allows specifying a similar behaviour, as it forces the total amount of offered power to be accepted, otherwise, no power will be sold. This is similar to the fillor-kill condition of the block orders. The inference on the information contained in the ontology allows players to use similar behaviours in different markets, taking advantage on the opportunities and particularities of each market.

Besides the fundamental role of the developed ontologies for the possibility of conducting this type of studies, the restructuring process of MASCEM has also enabled the inclusion of different types of markets, with different characteristics and particularities. Moreover, the four presented scenarios have been executed simultaneously, which was not possible before MASCEM's restructuring. Using the restructured simulator and making use of its novel ontologies, it is now possible to undertake advanced simulation studies, including different market types and players from very different natures.

\section{Conclusions}

In order to disseminate the development of interoperable MAS within power engineering, interconnection issues must be addressed. There is a growing need for knowledge exchange with the aim at providing full interoperability between different systems to take full advantage of these systems. The Electricity Markets Ontology (EMO) is proposed, with the aim of overcoming these issues, gathering the EM main concepts, enabling the interoperability of independently developed multi-agent based simulation platforms.

Additionally, particular modules conceived to deal with the different communications between market operators and market players have also been developed, namely: (i) the Call For Proposal Ontology (CFP) and (ii) the Electricity Markets Results Ontology (EMR).

Using EMO, different types of agents are able to communicate with each other, understanding a common language, while providing the means for any agent from external systems to do the same, simply by importing the developed ontologies. By "speaking the same language”, agents from different communities can understand each other and communicate efficiently, without the need for spending unnecessary computational resources and execution time in translating messages, which is an essential issue in a simulation process.

The developed ontologies are publicly available online so it can be easily accessed, reused and extended by Ontology Engineers or MAS developers in the scope of EM. This is a relevant contribution, not only to provide the participation in joint simulations with MASCEM, but also to give the basis for the development of other systems specific ontologies. The comparison of the system's performance with and 
without the use of ontologies is considered as future work, as well as the conversion of RDF messages to JSON-LD ${ }^{9}$ in order to reduce the computational weight of the communications.

The presented case study has proven the usefulness and advantages of using the proposed ontology in the scope of the wholesale EM. It showed how the use of the proposed ontology allows players to participate in different market types, including markets that players are not familiar with.

The new EM simulator resulting from the integration of the proposed ontology in MASCEM provides a solid platform to study and explore the implications and consequences of new and already existing approaches in EM. Researchers of the power systems area consider tools with this type of capabilities essential in order to be prepared to deal with the constant changes in the EM environment. It is important to point out that it was used real EM players' data in the simulation, extracted from the market operator's website.

\section{References}

ACER - Agency for the Cooperation of Energy Regulators, Electricity $>$ Regional initiatives $>$ Cross Regional Roadmaps $>$ CrossBorder Intraday, http://www.acer.europa.eu/en/Electricity/Regional_initiatives/Cross_Regional_Roadmaps/Pages/2.-Crossborder-Intraday.aspx [accessed on July 2016].

ACER - Agency for the Cooperation of Energy Regulators, Electricity $>$ Regional initiatives $>$ Cross Regional Roadmaps $>$ Market Coupling, http://www.acer.europa.eu/en/Electricity/Regional_initiatives/Cross_Regional_Roadmaps/Pages/1.-MarketCoupling.aspx [accessed on July 2016].

ACER report, "Regional Initiatives Status Review Report 2015", http://www.acer.europa.eu/Official_documents/Acts_of_the_Agency/Publication/ACER\%20Regional\%20Initiatives\%20St atus\%20Review\%20Report\%202015.pdf [accessed on July 2016].

Alexopoulos P., Kafentzis K., Zoumas C. ELMO: An Interoperability Ontology for the Electricity Market. Proceedings of the International Conference on e-Business, Milan, Italy, July 7-10, 2009.

Alvarado-Pérez , J. C., Peluffo-Ordóñez, D. H., Therón, R., Bridging The Gap Between Human Knowledge And Machine Learning, Advances In Distributed Computing And Artificial Intelligence Journal, Salamanca University Press Journal, vol. 4, no.1, 2015.

CAISO - California Independent System Operator, homepage, http://www.caiso.com [accessed on July 2016].

Catterson V. et al. An upper ontology for power engineering applications. April 2010. [Online]. Available: http://sites.ieee.org/pesmas/, accessed on July 2016.

Catterson V., 2006. Engineering Robustness, Flexibility, and Accuracy into a Multi-Agent System for Transformer Condition Monitoring. Doctoral thesis, University of Strathclyde, 2006.

Cincotti, S.; Gallo, G. Genoa Artificial Power-Exchange. Agents Artificial Intelligence, 2013. Springer Berlin Heidelberg 2013:34863. http://dx.doi.org/10.1007/978-3-642-36907-0_23.

Dai W., Dubinin V. and Vyatkin V. Automatically Generated Layered Ontological Models for Semantic Analysis of ComponentBased Control Systems. IEEE Transactions on Industrial Informatics, vol.9, issue 4, pp. 2124-2136, 2013.

Dam K. and Chapping E. Coupling agent-based models of natural gas and electricity markets. In Proceedings of the First International Workshop on Agent Technologies for Energy Systems (ATES 2010), pages 45-52, 11 May 2010.

\footnotetext{
${ }^{9}$ http://json-Id.org/
}

Advances in Distributed Computing and

Artificial Intelligence Journal

(C) Ediciones Universidad de Salamanca / cc by-nc-nd
ADCAIJ, Regular Issue, Vol.5 N.2 (2016)

http://adcaij.usal.es 
Dam K. and Keirstead J. Re-use of an ontology for modelling urban energy systems. In Proceedings of the 3rd International Conference on Infrastructure Systems and Services: Next Generation Infrastructure Systems for Eco-Cities (INFRA), Shenzhen, China, 11-13 November 2010.

EPEXSPOT - European Power Exchange, 2016. [Online]. Homepage: https://www.epexspot.com/, accessed on July 2016.

EPEXSPOT - European Power Exchange, Products, Day-Ahead Auction, 2015. [Online]. Available: https://www.epexspot.com/en/product-info/auction, accessed on July 2016.

EUPHEMIA Public Description, PCR Market Coupling Algorithm, January 2016, available: https://www.epexspot.com/document/34460/Euphemia\%3A\%20Public\%20documentation\%20-\%20January\%202016 [accessed on July, 2016].

Ferber J. Multi-Agent system: an introduction to distributed artificial intelligence. Harlow: Addison Wesley Longman; 1999.

Foundation for Intelligent Physical Agents (FIPA), ACL Message Structure Specification, 2002. [Online]. Available: http://www.fipa.org/specs/fipa00061/, accessed on July 2016

Foundation for Intelligent Physical Agents (FIPA), FIPA Agent Management Specification, 2004. [Online]. Available: http://fipa.org/specs/fipa00023/, accessed on July 2016.

Foundation for Intelligent Physical Agents (FIPA), FIPA Ontology Service Specification, 2001. [Online]. Available: http://www.fipa.org/specs/fipa00086/XC00086D.html, accessed on September 2015.

Frikha, M., Mhiri, M., Gargour, F. A Semantic Social Recommender System Using Ontologies Based Approach For Tunisian Tourism. Advances In Distributed Computing And Artificial Intelligence Journal, Salamanca University Press Journal, vol. 4, no.1, 2015.

Gestori Mercati Energetici, homepage, 2016. [Online]. Available: http://www.mercatoelettrico.org/En/Default.aspx, accessed on July 2016.

Koritarov, V. Real-World Market Representation with Agents: Modeling the Electricity Market as a Complex Adaptive System with an Agent-Based Approach. IEEE Power \& Energy magazine, pp. 39-46, 2004.

Li, H.; Tesfatsion, L. Development of Open Source Software for Power Market Research: The AMES Test Bed. Journal of Energy Markets, vol. 2, no. 2, pp. 111-128, 2009.

McArthur S.; et al. Multi-agent systems for power engineering applications - part ii: Technologies, standards, and tools for building multi-agent systems. Power Systems, IEEE Transactions on, 2007.

Meeus L, Purchalaa K, Belmans R. Development of the internal electricity market in Europe. Electr J 2005;18(6):25-35.

MIBEL - Mercado Ibérico de Electricidade, 2016. [Online]. Homepage: http://www.mibel.com/, accessed on July 2016.

MISO Energy, homepage, http://www.misoenergy.org [accessed on July 2016].

Morais, H.; et al. SOSPO-SP: secure operation of sustainable power systems simulation platform for real-time system state evaluation and control. IEEE Trans Ind Inf 2014:2318-29.

Pinto, T.; Morais, H.; Sousa, T.M.; Sousa, T.; Vale, Z.; Praça, I.; Faia, R.; Pires, E.J.S. Adaptive Portfolio Optimization for Multiple Electricity Markets Participation. IEEE Transactions on Neural Networks and Learning Systems, vol. 27, no.8, pp. 17201733, August 2016.

Pinto, T.; Vale, Z.; Rodrigues, F.; Morais, H.; Praça, I. Bid definition method for electricity markets based on an adaptive multiagent system. Advances on Practical Applications of Agents and Multiagent Systems, 309-316, 2011.

Pinto, T.; Vale, Z.; Sousa, T.M. and Praça, I. Negotiation Context Analysis in Electricity Markets. Energy, Elsevier, vol. 85, pp. 7893, June 2015.

Nord Pool Spot - Trading, Day-ahead market Elspot, 2016 [Online]. Available: http://www.nordpoolspot.com/TAS/Day-aheadmarket-Elspot/, accessed on July 2016

Nord Pool Spot, 2016 [Online]. Homepage: http://www.nordpoolspot.com/, accessed on July 2016.

Advances in Distributed Computing and

Artificial Intelligence Journal

(C)Ediciones Universidad de Salamanca / cc by-nc-nd
ADCAIJ, Regular Issue, Vol.5 N.2 (2016)

http://adcaij.usal.es 
OMIE, Markets and Products, Electricity Market, About our Market, available: http://www.omie.es/en/home/markets-andproducts/about-our-market, accessed on July 2016.

ONS - Operador Nacional do Sistema Elétrico (Electrical System Nacional Operator), homepage, http://www.ons.org.br [accessed on July 2016].

PCR report, "EUPHEMIA: Description and functioning", May 2014, available: https://www.mercatoelettrico.org/en/MenuBiblioteca/Documenti/20140704EuphemiaPublicDocumentation.pdf [accessed on July 2016].

PCR report, "ITALIAN BORDERS SUCCESSFULLY COUPLED", February, 2015, available: http://m.omie.es/files/2015-0224_MRC_PCR_IBWT_Communication_SuccessfulGoLive.pdf?m=yes [accessed on July, 2016].

Santos G., Pinto T., Morais H., Praça I. and Vale Z. Complex Market integration in MASCEM electricity market simulator. International Conference on the European Energy Market 11 - EEM, 2011.

Santos, G. et al. Multi-Agent Simulation of Competitive Electricity Markets: Autonomous systems cooperation for European Market modelling. Energy Conversion and Management, 99, 387-399, July 2015.

Santos, G.; Pinto, T.; Praça, I. and Vale Z. MASCEM: Optimizing the performance of a multi-agent system. Energy, vol. 111, pp. 513-524, September 2016.

Shahidehpour M, Yamin H, Li Z. Market operations in electric power systems: forecasting, scheduling, and risk management”. WileyIEEE Press; 2002. p. 233-74.

Sharma, K.C.; Bhakar, R.; Tiwari, H.P. Strategic bidding for wind power producers in electricity markets. Energy Conversion and Management 2014, 86, 259-267, DOI: 10.1016/j.enconman.2014.05.002. Available online: http://www.sciencedirect.com/science/article/pii/S0196890414004129 (accessed on July 2016).

Sioshansi, F.P. “Evolution of Global Electricity Markets - New paradigms, new challenges, new approaches”, Academic Press, 2013.

Vrba, P.; et al. A review of agent and service-oriented concepts applied to intelligent energy systems. IEEE Trans Ind Inf 2014;10(3):1890-903

Wu J., Guan X., Gao F. and Sun G. Social welfare maximization auction for electricity markets with elastic demand. 7th World Congress on Intelligent Control and Automation, pp. 7157-7162, 25-27 June 2008. 